\title{
The $\mu-\tau$ reflection symmetry of Dirac neutrinos and its breaking effect via quantum corrections
}

\author{
Zhi-zhong Xing, ${ }^{a, b}$ Di Zhang ${ }^{a}$ and Jing-yu Zhu ${ }^{a}$ \\ ${ }^{a}$ Theoretical Physics Division, Institute of High Energy Physics and \\ School of Physical Sciences, University of Chinese Academy of Sciences, \\ 19B Yuquan Road, Shijingshan District, Beijing 100049, China \\ ${ }^{b}$ Center for High Energy Physics, Peking University, \\ No. 5 Yiheyuan Road, Haidian District, Beijing 100080, China \\ E-mail: xingzz@ihep.ac.cn, dizhang@mails.ccnu.edu.cn, \\ zhujingyu@ihep.ac.cn
}

Abstract: Given the Dirac neutrino mass term, we explore the constraint conditions which allow the corresponding mass matrix to be invariant under the $\mu$ - $\tau$ reflection transformation, leading us to the phenomenologically favored predictions $\theta_{23}=\pi / 4$ and $\delta=3 \pi / 2$ in the standard parametrization of the $3 \times 3$ lepton flavor mixing matrix. If such a flavor symmetry is realized at a superhigh energy scale $\Lambda_{\mu \tau}$, we investigate how it is spontaneously broken via the one-loop renormalization-group equations (RGEs) running from $\Lambda_{\mu \tau}$ down to the Fermi scale $\Lambda_{\mathrm{F}}$. Such quantum corrections to the neutrino masses and flavor mixing parameters are derived, and an analytical link is established between the Jarlskog invariants of CP violation at $\Lambda_{\mu \tau}$ and $\Lambda_{\mathrm{F}}$. Some numerical examples are also presented in both the minimal supersymmetric standard model and the type-II two-Higgs-doublet model, to illustrate how the octant of $\theta_{23}$, the quadrant of $\delta$ and the neutrino mass ordering are correlated with one another as a result of the RGE-induced $\mu-\tau$ reflection symmetry breaking effects.

KeYwords: Beyond Standard Model, Neutrino Physics

ARXIV EPRINT: 1708.09144 


\section{Contents}

1 Introduction 1

$\begin{array}{lll}2 & \mu-\tau \text { reflection symmetry } & 3\end{array}$

3 RGE corrections to $H_{\nu} \quad 6$

4 RGE corrections to $U$

$\begin{array}{lll}5 & \text { Summary } & 16\end{array}$

\section{Introduction}

The discoveries of solar, atmospheric, reactor and accelerator neutrino oscillations [1] have demonstrated that the standard model (SM) of electroweak interactions is incomplete and must be extended in a proper way so as to accommodate tiny neutrino masses and significant lepton flavor mixing. The simplest way to do so is to introduce three right-handed (or $\mathrm{SU}(2)$-singlet) neutrino fields $N_{\alpha \mathrm{R}}$ (for $\left.\alpha=e, \mu, \tau\right)$ into the $\mathrm{SM}$ and write out a gaugeinvariant, Lorentz-invariant and lepton-number-conserving mass term of the form

$$
-\mathcal{L}_{\text {Dirac }}=\overline{\ell_{\mathrm{L}}} Y_{\nu} \tilde{H} N_{\mathrm{R}}+\text { h.c. },
$$

where $\tilde{H}=\mathrm{i} \sigma_{2} H^{*}$ with $H$ being the SM Higgs doublet, $\ell_{\mathrm{L}}$ denotes the left-handed lepton doublet column vector, and $N_{\mathrm{R}}$ represents the right-handed neutrino column vector with the $N_{\alpha \mathrm{R}}$ components. After spontaneous gauge symmetry breaking, the above Dirac neutrino mass term turns out to be ${ }^{1}$

$$
-\mathcal{L}_{\text {Dirac }}^{\prime}=\overline{\nu_{\mathrm{L}}} M_{\nu} N_{\mathrm{R}}+\text { h.c. },
$$

where $M_{\nu}=Y_{\nu}\langle H\rangle$ with $\langle H\rangle=v / \sqrt{2}$ and $v \simeq 246 \mathrm{GeV}$. The three neutrino masses $m_{i}$ (for $i=1,2,3)$ can therefore be achieved from diagonalizing $M_{\nu}$ if its texture is specified in a given model, but the smallness of $m_{i}$ is not really explained in this manner. While many theorists believe that the neutrinos should be Majorana fermions [4], by which their small

\footnotetext{
${ }^{1}$ If the minimal supersymmetric standard model (MSSM) is concerned, the charged-lepton and neutrino sectors are associated with the Higgs doublets $H_{1}$ (with the hypercharge $+1 / 2$ and the vacuum expectation value $v \cos \beta / \sqrt{2}$ ) and $H_{2}$ (with the hypercharge $-1 / 2$ and the vacuum expectation value $v \sin \beta / \sqrt{2}$ ), respectively (see e.g. [2] and references therein). But for the type-II two-Higgs-doublet model (2HDM), the Higgs doublet $H_{1}$ is coupled to both the charged-lepton and neutrino sectors (for a review with extensive references see ref. [3]). These two interesting scenarios will be used to illustrate quantum corrections to the $\mu-\tau$ reflection symmetry in section 4 .
} 
masses can be naturally understood via a seesaw mechanism, ${ }^{2,3}$ the simplicity of the Dirac neutrino mass generation mechanism $d o$ attract quite a lot of attention. ${ }^{4,5}$ Before the Majorana nature of massive neutrinos is ultimately determined with the help of a measurement of the neutrinoless double-beta decay or other lepton-number-violating processes [40], ${ }^{6}$ it makes sense to study the phenomenology of Dirac neutrinos as well.

Assuming the massive neutrinos to be the Dirac fermions, we shall begin with eq. (1.2) to explore the $\mu-\tau$ reflection symmetry of $\mathcal{L}_{\text {Dirac }}^{\prime}$ and the resulting texture of $M_{\nu}$ in the basis where the flavor eigenstates of three charged leptons are identified with their mass eigenstates. The motivation for this study is simply because such a flavor symmetry may naturally lead us to the phenomenologically favored predictions $\theta_{23}=\pi / 4$ and $\delta=3 \pi / 2$ in the standard parametrization of the $3 \times 3$ Pontecorvo-Maki-Nakagawa-Sakata (PMNS) lepton flavor mixing matrix $U[42,43]$ that is used to diagonalize $M_{\nu} M_{\nu}^{\dagger}$. Provided the $\mu-\tau$ reflection symmetry is realized at a superhigh energy scale $\Lambda_{\mu \tau}$, we shall investigate how it is spontaneously broken due to the running of $M_{\nu}$ from $\Lambda_{\mu \tau}$ down to the Fermi scale $\Lambda_{\mathrm{F}} \sim v \sim$ $10^{2} \mathrm{GeV}$ through the one-loop renormalization-group equations (RGEs) in the framework of either the MSSM or the type-II 2HDM. Such quantum corrections to the three neutrino masses and four flavor mixing parameters will be derived, and an analytical link will be established between the Jarlskog invariants of leptonic CP violation at $\Lambda_{\mu \tau}$ and $\Lambda_{\mathrm{F}}$. We shall also present some numerical examples in both the MSSM and the type-II 2HDM to illustrate how the octant of $\theta_{23}$, the quadrant of $\delta$ and the neutrino mass ordering are correlated with one another as a result of the RGE-triggered $\mu-\tau$ reflection symmetry breaking effects.

The content of this work is new in several aspects. First, applying the $\mu-\tau$ reflection symmetry to the Dirac neutrino mass term, in which $M_{\nu}$ is in general neither symmetric nor Hermitian, has not been tried before. Second, the integral form of the RGE corrections to $M_{\nu}$ is derived for the first time, so is the integral form of the RGE effects on the neutrino masses and flavor mixing parameters. Third, a concise analytical relationship between the Jarlskog invariants of CP violation at $\Lambda_{\mu \tau}$ and $\Lambda_{\mathrm{F}}$ is derived for the first time. Fourth, a comparison is made between the MSSM and the type-II 2HDM, which leads to the opposite deviations of $\theta_{12}, \theta_{13}, \theta_{23}$ and $\delta$ from their corresponding values in the $\mu-\tau$ reflection symmetry limit.

The remaining parts of this paper are organized as follows. In section 2 we shall find out the constraint conditions which allow the Dirac neutrino mass matrix $M_{\nu}$ to be invariant under the $\mu-\tau$ reflection transformation. Section 3 is devoted to the derivation of the integral form of the RGE corrections to $M_{\nu} M_{\nu}^{\dagger}$ when it runs from $\Lambda_{\mu \tau}$ down to $\Lambda_{\mathrm{F}}$, and to the derivation of an analytical relationship between the Jarlskog invariants at $\Lambda_{\mu \tau}$ and $\Lambda_{\mathrm{F}}$. In section 4 we calculate the RGE-induced corrections to the neutrino masses and flavor mixing parameters in a perturbation way, and illustrate their salient features by taking a few numerical examples in both the MSSM and the type-II 2HDM. Finally, we summarize our main results and make a conclusion in section 5 .

\footnotetext{
${ }^{2}$ The canonical seesaw mechanism was originally proposed in refs. [5-9].

${ }^{3}$ For a brief review of other seesaw mechanisms see refs. [10, 11].

${ }^{4}$ See e.g. refs. [12-24].

${ }^{5}$ For recent works see e.g. refs. [25-39].

${ }^{6}$ For a recent review of this topic see ref. [41] and references therein.
} 


\section{$2 \mu-\tau$ reflection symmetry}

Given the Dirac neutrino mass term in eq. (1.2), let us consider the following transformations of the six neutrino fields: ${ }^{7}$

$$
\begin{array}{ll}
\nu_{e \mathrm{~L}} \leftrightarrow \nu_{e \mathrm{~L}}^{\mathrm{c}}, & N_{e \mathrm{R}} \leftrightarrow N_{e \mathrm{R}}^{\mathrm{c}}, \\
\nu_{\mu \mathrm{L}} \leftrightarrow \nu_{\tau \mathrm{L}}^{\mathrm{c}}, & N_{\mu \mathrm{R}} \leftrightarrow N_{\tau \mathrm{R}}^{\mathrm{c}}, \\
\nu_{\tau \mathrm{L}} \leftrightarrow \nu_{\mu \mathrm{L}}^{\mathrm{c}}, & N_{\tau \mathrm{R}} \leftrightarrow N_{\mu \mathrm{R}}^{\mathrm{c}},
\end{array}
$$

where $\nu_{\alpha \mathrm{L}}^{\mathrm{c}} \equiv \mathcal{C}{\overline{\nu_{\alpha \mathrm{L}}}}^{\mathrm{T}}$ and $N_{\alpha \mathrm{L}}^{\mathrm{c}} \equiv \mathcal{C}{\overline{N_{\alpha \mathrm{L}}}}^{\mathrm{T}}$ (for $\left.\alpha=e, \mu, \tau\right)$ with $\mathrm{T}$ denoting the transpose and $\mathcal{C}$ being the charge-conjugation operator and satisfying $\mathcal{C}^{-1}=\mathcal{C}^{\dagger}=\mathcal{C}^{\mathrm{T}}=-\mathcal{C}$ [46]. Under such transformations, eq. (1.2) turns out to be

$$
\begin{aligned}
-\mathcal{L}_{\text {Dirac }}^{\prime} & =\overline{\nu_{\mathrm{L}}^{\mathrm{c}}} S M_{\nu} S N_{\mathrm{R}}^{\mathrm{c}}+\overline{N_{\mathrm{R}}^{\mathrm{c}}} S M_{\nu}^{\dagger} S \nu_{\mathrm{L}}^{\mathrm{c}} \\
& =-\nu_{\mathrm{L}}^{\mathrm{T}} S M_{\nu} S{\overline{N_{\mathrm{R}}}}^{\mathrm{T}}-N_{\mathrm{R}}^{\mathrm{T}} S M_{\nu}^{\dagger} S{\overline{\nu_{\mathrm{L}}}}^{\mathrm{T}} \\
& =\overline{\nu_{\mathrm{L}}} S M_{\nu}^{*} S N_{\mathrm{R}}+\overline{N_{\mathrm{R}}} S M_{\nu}^{\mathrm{T}} S \nu_{\mathrm{L}},
\end{aligned}
$$

in which the property of $\mathcal{L}^{\prime}$ as a Lorentz scalar has been used, and

$$
S=\left(\begin{array}{lll}
1 & 0 & 0 \\
0 & 0 & 1 \\
0 & 1 & 0
\end{array}\right)
$$

If $\mathcal{L}^{\prime}$ is required to be invariant under the above $\mu-\tau$ reflection transformations [47], then the Dirac neutrino mass matrix

$$
M_{\nu} \equiv\left(\begin{array}{ccc}
\langle m\rangle_{e e} & \langle m\rangle_{e \mu} & \langle m\rangle_{e \tau} \\
\langle m\rangle_{\mu e} & \langle m\rangle_{\mu \mu} & \langle m\rangle_{\mu \tau} \\
\langle m\rangle_{\tau e} & \langle m\rangle_{\tau \mu} & \langle m\rangle_{\tau \tau}
\end{array}\right)
$$

must satisfy the relationship

$$
M_{\nu}=S M_{\nu}^{*} S
$$

In other words, the elements of $M_{\nu}$ must satisfy

$$
\begin{array}{ll}
\langle m\rangle_{e e}=\langle m\rangle_{e e}^{*}, & \langle m\rangle_{e \mu}=\langle m\rangle_{e \tau}^{*}, \\
\langle m\rangle_{\mu e}=\langle m\rangle_{\tau e}^{*}, & \langle m\rangle_{\mu \tau}=\langle m\rangle_{\tau \mu}^{*}, \\
\langle m\rangle_{\mu \mu}=\langle m\rangle_{\tau \tau}^{*} . &
\end{array}
$$

\footnotetext{
${ }^{7}$ In this work we focus on a possible $\mu-\tau$ reflection symmetry of the Dirac neutrino mass matrix after spontaneous gauge symmetry breaking. Otherwise, the neutrino field transformations made in eq. (2.1) would affect some other parts of the Lagrangian of the electroweak interactions. To build a consistent lepton mass model with the $\mu-\tau$ flavor symmetry in the neutrino sector instead of the charged-lepton sector, one should introduce some extra scalar fields coupling to the two sectors in a different way (see e.g. refs. $[44,45])$. But here we simply assume that the $\mu-\tau$ reflection symmetry does not apply to the charged-lepton sector. In this sense the invariance of $\mathcal{L}_{\text {Dirac }}^{\prime}$ under the transformations in eq. (2.1) can just serve as a phenomenological guiding principle to obtain the special texture of $M_{\nu}$ in eq. (2.7).
} 
Then the texture of $M_{\nu}$ can be simply parametrized as

$$
M_{\nu}=\left(\begin{array}{ccc}
a & b & b^{*} \\
e & c & d \\
e^{*} & d^{*} & c^{*}
\end{array}\right)
$$

where $a$ is real, and the other four parameters are in general complex. To diagonalize $M_{\nu}$ in eq. (2.7), one may do a bi-unitary transformation of the form

$$
U^{\dagger} M_{\nu} Q=\hat{M}_{\nu}
$$

where $U$ and $Q$ are the unitary matrices, and $\hat{M}_{\nu} \equiv \operatorname{Diag}\left\{m_{1}, m_{2}, m_{3}\right\}$ with $m_{i}$ (for $i=$ $1,2,3)$ being the neutrino masses. In the basis where the flavor eigenstates of three charged leptons are identified with their mass eigenstates, the unitary matrix $U$ is just the PMNS flavor mixing matrix which manifests itself in the leptonic weak charged-current interactions.

It proves more convenient to consider the Hermitian matrix

$$
\begin{aligned}
H_{\nu} & \equiv M_{\nu} M_{\nu}^{\dagger}=U \hat{M}_{\nu}^{2} U^{\dagger} \\
& =\left(\begin{array}{ccc}
A & B & B^{*} \\
B^{*} & C & D \\
B & D^{*} & C
\end{array}\right),
\end{aligned}
$$

where

$$
\begin{aligned}
& A=a^{2}+2|b|^{2}, \\
& B=a e^{*}+b c^{*}+b^{*} d^{*}, \\
& C=|e|^{2}+|c|^{2}+|d|^{2}, \\
& D=e^{2}+2 c d .
\end{aligned}
$$

Moreover, let us parametrize $U$ as $U \equiv P V$, where $P=\operatorname{Diag}\left\{e^{\mathrm{i} \phi_{e}}, e^{\mathrm{i} \phi_{\mu}}, e^{\mathrm{i} \phi_{\tau}}\right\}$ is an unphysical phase matrix associated with the charged-lepton fields, ${ }^{8}$ and

$$
V=\left(\begin{array}{ccc}
c_{12} c_{13} & s_{12} c_{13} & s_{13} e^{-\mathrm{i} \delta} \\
-s_{12} c_{23}-c_{12} s_{13} s_{23} e^{\mathrm{i} \delta} & c_{12} c_{23}-s_{12} s_{13} s_{23} e^{\mathrm{i} \delta} & c_{13} s_{23} \\
-s_{12} s_{23}+c_{12} s_{13} c_{23} e^{\mathrm{i} \delta} & c_{12} s_{23}+s_{12} s_{13} c_{23} e^{\mathrm{i} \delta} & -c_{13} c_{23}
\end{array}\right)
$$

with $c_{i j} \equiv \cos \theta_{i j}$ and $s_{i j} \equiv \sin \theta_{i j}$ (for $i j=12,13,23$ ). At a given energy scale, one may rotate away $P$ and then express the four flavor mixing parameters of $V$ in terms of the elements of

$$
\begin{aligned}
\bar{H}_{\nu} & \equiv P^{\dagger} H_{\nu} P=V \hat{M}_{\nu}^{2} V^{\dagger} \\
& =\left(\begin{array}{ccc}
A & \bar{B} & \bar{B}^{*} \\
\bar{B}^{*} & C & \bar{D} \\
\bar{B} & \bar{D}^{*} & C
\end{array}\right),
\end{aligned}
$$

\footnotetext{
${ }^{8}$ Note that these unphysical phases should not be ignored in the course of deriving the RGEs of the neutrino masses and flavor mixing parameters, as one can see in section 4 . When using $U=P V$ to reconstruct the texture of $H_{\nu}$, we find that $\phi_{\mu}+\phi_{\tau}=2 \phi_{e}$ must be satisfied, as required by the $\mu-\tau$ reflection symmetry.
} 
where $\bar{B}=B e^{\mathrm{i}\left(\phi_{\mu}-\phi_{e}\right)}$ and $\bar{D}=D e^{\mathrm{i}\left(\phi_{\tau}-\phi_{\mu}\right)}$. In this way the unphysical phases hidden in $B$ and $D$ will be cancelled by $\phi_{\mu}-\phi_{e}$ and $\phi_{\tau}-\phi_{\mu}$, respectively. Then we do a similar diagonalization of $\bar{H}_{\nu}$ as that done in refs. [48-50] (and references therein) and obtain

$$
\begin{aligned}
& \theta_{12}=\frac{1}{2} \arctan \left[2 \frac{|\operatorname{Re} \bar{B}| \sqrt{2(\operatorname{Re} \bar{B})^{2}+(\operatorname{Im} \bar{D})^{2}}}{|\operatorname{Im} \bar{B} \operatorname{Im} \bar{D}-2 \operatorname{Re} \bar{B} \operatorname{Re} \bar{D}|}\right], \\
& \theta_{13}=\arctan \left[\frac{1}{\sqrt{2}}\left|\frac{\operatorname{Im} \bar{D}}{\operatorname{Re} \mid}\right|\right]
\end{aligned}
$$

together with the typical predictions

$$
\theta_{23}=\frac{\pi}{4}, \quad \delta=\frac{\pi}{2} \quad \text { or } \quad \frac{3 \pi}{2} .
$$

These two numerical predictions, which have been well known for the Majorana neutrino mass matrix with the $\mu-\tau$ reflection symmetry, ${ }^{9}$ are now achieved in the Dirac case with the same flavor symmetry. It is easy to see that eq. (2.14) leads us to the equalities

$$
\left|V_{\mu 1}\right|=\left|V_{\tau 1}\right|, \quad\left|V_{\mu 2}\right|=\left|V_{\tau 2}\right|, \quad\left|V_{\mu 3}\right|=\left|V_{\tau 3}\right|,
$$

which are sometimes referred to as the $\mu-\tau$ reflection symmetry at the PMNS matrix level. One may therefore define the asymmetries $\mathcal{A}_{i} \equiv\left|V_{\mu i}\right|^{2}-\left|V_{\tau i}\right|^{2}$ (for $i=1,2,3$ ) to measure the effects of $\mu-\tau$ symmetry breaking in a rephasing-invariant way [52].

Of course, it is more fundamental to understand how the $\mu$ - $\tau$ reflection symmetry of $M_{\nu}$ or $H_{\nu}$ can be spontaneously or explicitly broken, both for the model-building purpose and for explaining currently available neutrino oscillation data [53]. Following the discussions about the $\mu-\tau$ symmetry breaking of the Majorana neutrino mass matrix [51, 54-57], one can similarly introduce the most general perturbation to the Dirac neutrino mass matrix with the $\mu-\tau$ reflection symmetry. But we find that it is more convenient to focus on the perturbation to $H_{\nu}$ in eq. (2.9) instead of $M_{\nu}$ in eq. (2.7), simply because the former is always Hermitian. In this case the perturbation matrix $\Delta H_{\nu}$ can also be arranged to be Hermitian, and it can be decomposed into two parts: one part conserves the original $\mu-\tau$ reflection symmetry and the other part violates this symmetry. Namely,

$$
\begin{aligned}
\Delta H_{\nu}=\left(\begin{array}{ccc}
\delta_{e e} & \delta_{e \mu} & \delta_{e \tau} \\
\delta_{e \mu}^{*} & \delta_{\mu \mu} & \delta_{\mu \tau} \\
\delta_{e \tau}^{*} & \delta_{\mu \tau}^{*} & \delta_{\tau \tau}
\end{array}\right)= & \frac{1}{2}\left(\begin{array}{ccc}
2 \delta_{e e} & \delta_{e \mu}+\delta_{e \tau}^{*} & \delta_{e \mu}^{*}+\delta_{e \tau} \\
\delta_{e \mu}^{*}+\delta_{e \tau} & \delta_{\mu \mu}+\delta_{\tau \tau} & 2 \delta_{\mu \tau} \\
\delta_{e \mu}+\delta_{e \tau}^{*} & 2 \delta_{\mu \tau}^{*} & \delta_{\mu \mu}+\delta_{\tau \tau}
\end{array}\right) \\
+ & \frac{1}{2}\left(\begin{array}{ccc}
0 & \delta_{e \mu}-\delta_{e \tau}^{*} & \delta_{e \tau}-\delta_{e \mu}^{*} \\
\delta_{e \mu}^{*}-\delta_{e \tau} & \delta_{\mu \mu}-\delta_{\tau \tau} & \mathbf{0} \\
\delta_{e \tau}^{*}-\delta_{e \mu} & \mathbf{0} & \delta_{\tau \tau}-\delta_{\mu \mu}
\end{array}\right),
\end{aligned}
$$

where $\delta_{e e}, \delta_{\mu \mu}$ and $\delta_{\tau \tau}$ are real, and all the parameters are expected to be reasonably small in magnitude. Because the symmetry-conserving part can be absorbed into $H_{\nu}$ via

\footnotetext{
${ }^{9}$ For the latest review with many references see ref. [51].
} 
a redefinition of its initial matrix elements, we are then left with

$$
H_{\nu}^{\prime}=H_{\nu}+\Delta H_{\nu}=\left(\begin{array}{ccc}
A^{\prime} & B^{\prime}\left(1+\epsilon_{1}\right) & B^{\prime *}\left(1-\epsilon_{1}^{*}\right) \\
B^{\prime *}\left(1+\epsilon_{1}^{*}\right) & C^{\prime}\left(1+\epsilon_{2}\right) & D^{\prime} \\
B^{\prime}\left(1-\epsilon_{1}\right) & D^{\prime *} & C^{\prime}\left(1-\epsilon_{2}\right)
\end{array}\right),
$$

where

$$
\begin{array}{ll}
A^{\prime}=A+\delta_{e e}, & B^{\prime}=B+\frac{\delta_{e \mu}+\delta_{e \tau}^{*}}{2}, \\
C^{\prime}=C+\frac{\delta_{\mu \mu}+\delta_{\tau \tau}}{2}, & D^{\prime}=D+\delta_{\mu \tau}
\end{array}
$$

and

$$
\epsilon_{1}=\frac{\delta_{e \mu}-\delta_{e \tau}^{*}}{2 B^{\prime}}, \quad \epsilon_{2}=\frac{\delta_{\mu \mu}-\delta_{\tau \tau}}{2 C^{\prime}} .
$$

It is obvious that $\epsilon_{1}$ and $\epsilon_{2}$ are complex and real, respectively. These two dimensionless parameters will vanish, if $\Delta H_{\nu}$ respects the $\mu-\tau$ reflection symmetry.

Although the above formulism can provide us with a generic picture of the $\mu-\tau$ symmetry breaking, it has to be specified so as to see the explicit symmetry-breaking effects. In the following we shall assume that the $\mu-\tau$ reflection symmetry is realized at a superhigh energy scale $\Lambda_{\mu \tau}$, and examine its breaking at the Fermi scale $\Lambda_{\mathrm{F}}$ via the one-loop RGEs.

\section{RGE corrections to $H_{\nu}$}

From the point of view of model building, a specific flavor symmetry is usually realized at a superhigh energy scale where some fundamental new physics beyond the SM can naturally manifest itself. In this case the phenomenological consequences of such a flavor symmetry should be confronted with the low-energy experimental data by running the relevant physical quantities down to the Fermi scale $\Lambda_{\mathrm{F}}$ via the RGEs. In ref. [52] the one-loop RGEs of the $\mu-\tau$ asymmetries $\mathcal{A}_{i}$ of the PMNS matrix $U$ have been derived. Here we are going to derive the integral form of the RGE corrections to $M_{\nu}$ and $H_{\nu}$.

The differential form of the one-loop RGE for the Dirac neutrino mass matrix $M_{\nu}$ in the framework of the MSSM or the $2 \mathrm{HDM}$ is known as [58-64] ${ }^{10}$

$$
16 \pi^{2} \frac{\mathrm{d} M_{\nu}}{\mathrm{d} t}=\left[G+C_{\nu} Y_{\nu} Y_{\nu}^{\dagger}+C_{l} Y_{l} Y_{l}^{\dagger}\right] M_{\nu}
$$

where $t \equiv \ln \left(\Lambda / \Lambda_{\mu \tau}\right)$ with $\Lambda$ being a renormalization scale, $Y_{\nu}$ and $Y_{l}$ are the Yukawa coupling matrices of the neutrinos and charged leptons, respectively. Given the MSSM, one has $C_{\nu}=3, C_{l}=1$, and $G \simeq-0.6 g_{1}^{2}-3 g_{2}^{2}+3 y_{t}^{2}$ with $g_{1,2}$ being the gauge couplings and $y_{t}$ being the top-quark Yukawa coupling in the $y_{u}^{2} \ll y_{c}^{2} \ll y_{t}^{2}$ approximation. If the type-II $2 \mathrm{HDM}$ is taken into account, one has $C_{\nu}=3 / 2, C_{l}=-3 / 2$, and $G \simeq-0.45 g_{1}^{2}-$ $2.25 g_{2}^{2}+y_{\tau}^{2}+3 y_{b}^{2}$ with $y_{\tau}$ and $y_{b}$ being the tau-lepton and bottom-quark Yukawa couplings in the $y_{e}^{2} \ll y_{\mu}^{2} \ll y_{\tau}^{2}$ and $y_{d}^{2} \ll y_{s}^{2} \ll y_{b}^{2}$ approximations. Since the neutrino masses $m_{i}$

\footnotetext{
${ }^{10}$ For a recent review with extensive references see ref. [65].
} 
are extremely small as compared with their charged partners, it is very safe to neglect the $Y_{\nu} Y_{\nu}^{\dagger}$ term in eq. (3.1). In the basis that we have chosen (i.e., the mass eigenstates of three charged leptons are identified with their flavor eigenstates), $Y_{l} Y_{l}^{\dagger}=D_{l}^{2} \equiv \operatorname{Diag}\left\{y_{e}^{2}, y_{\mu}^{2}, y_{\tau}^{2}\right\}$ holds, where $y_{\alpha}^{2}=2\left(1+\tan ^{2} \beta\right) m_{\alpha}^{2} / v^{2}$ (for $\alpha=e, \mu, \tau$ ) with $\tan \beta$ being the ratio of the vacuum expectation value of $H_{2}$ to that of $H_{1}$ in the MSSM or the type-II 2HDM. Then eq. (3.1) leads us to the RGE of $H_{\nu}$ as follows:

$$
16 \pi^{2} \frac{\mathrm{d} H_{\nu}}{\mathrm{d} t}=2 G H_{\nu}+D_{l}^{2} H_{\nu}+H_{\nu} D_{l}^{2} .
$$

Integrating eq. (3.2) from $\Lambda_{\mu \tau}$ to $\Lambda_{\mathrm{F}}$, we immediately arrive at

$$
H_{\nu}^{\prime}=I_{G}^{2} T_{l} H_{\nu} T_{l},
$$

where $H_{\nu}$ and $H_{\nu}^{\prime}$ are associated respectively with the scales $\Lambda_{\mu \tau}$ and $\Lambda_{\mathrm{F}}, T_{l} \equiv$ $\operatorname{Diag}\left\{I_{e}, I_{\mu}, I_{\tau}\right\}$, and the evolution functions are

$$
\begin{aligned}
& I_{G}=\exp \left[\frac{1}{16 \pi^{2}} \int_{0}^{t^{\prime}} G \mathrm{~d} t\right], \\
& I_{\alpha}=\exp \left[\frac{C_{l}}{16 \pi^{2}} \int_{0}^{t^{\prime}} y_{\alpha}^{2} \mathrm{~d} t\right],
\end{aligned}
$$

where $t^{\prime} \equiv \ln \left(\Lambda_{\mathrm{F}} / \Lambda_{\mu \tau}\right)$, and $\alpha$ runs over $e, \mu$ and $\tau$. If one is more interested in the relationship between $M_{\nu}^{\prime}$ at $\Lambda_{\mathrm{F}}$ and $M_{\nu}$ at $\Lambda_{\mu \tau}$, then it is straightforward to obtain

$$
M_{\nu}^{\prime}=I_{G} T_{l} M_{\nu}
$$

either from integrating eq. (3.1) or from decomposing eq. (3.3).

Note that $y_{e}^{2} \ll y_{\mu}^{2} \ll y_{\tau}^{2} \lesssim 0.25$ holds at the Fermi scale $\Lambda_{\mathrm{F}}$ for $\tan \beta \lesssim 50$, and their values decrease as the energy scale grows up [66,67]. It is therefore an excellent approximation to take $T_{l} \simeq \mathbf{1}-\operatorname{Diag}\left\{0,0, \Delta_{\tau}\right\}$ with $\mathbf{1}$ being the $3 \times 3$ unitary matrix and

$$
\Delta_{\tau}=\frac{C_{l}}{16 \pi^{2}} \int_{t^{\prime}}^{0} y_{\tau}^{2} \mathrm{~d} t
$$

which is a small quantity of $\mathcal{O}(0.1)$ or much smaller. To illustrate, figure 1 shows the numerical changes of $I_{G}$ and $\Delta_{\tau}$ with the energy scale $\Lambda$ in the MSSM and the type-II 2HDM by fixing $\Lambda_{\mu \tau}=10^{14} \mathrm{GeV}$ as the initial point and taking $\tan \beta=10$ and 30 as two typical inputs. One can see that the signs of $\Delta_{\tau}$ are opposite in these two scenarios, and thus they are distinguishable at low energies. Now let us assume that the $\mu-\tau$ reflection symmetry of $M_{\nu}$ in eq. (2.7) or $H_{\nu}$ in eq. (2.9) is realized at $\Lambda_{\mu \tau}$. Then at the electroweak scale $\Lambda_{\mathrm{F}}$ we have

$$
H_{\nu}^{\prime} \simeq I_{G}^{2}\left[H_{\nu}-\Delta_{\tau}\left(\begin{array}{ccc}
0 & 0 & B^{*} \\
0 & 0 & D \\
B & D^{*} & 2 C
\end{array}\right)\right],
$$


MSSM

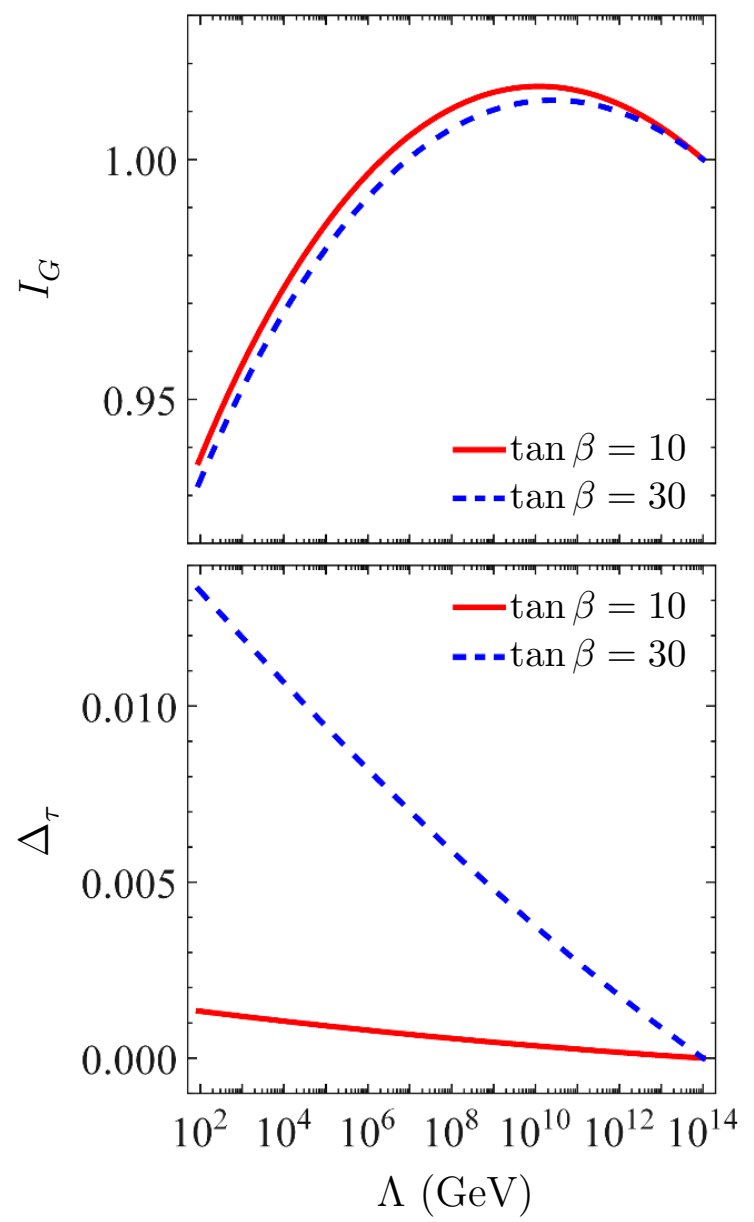

$2 \mathrm{HDM}$
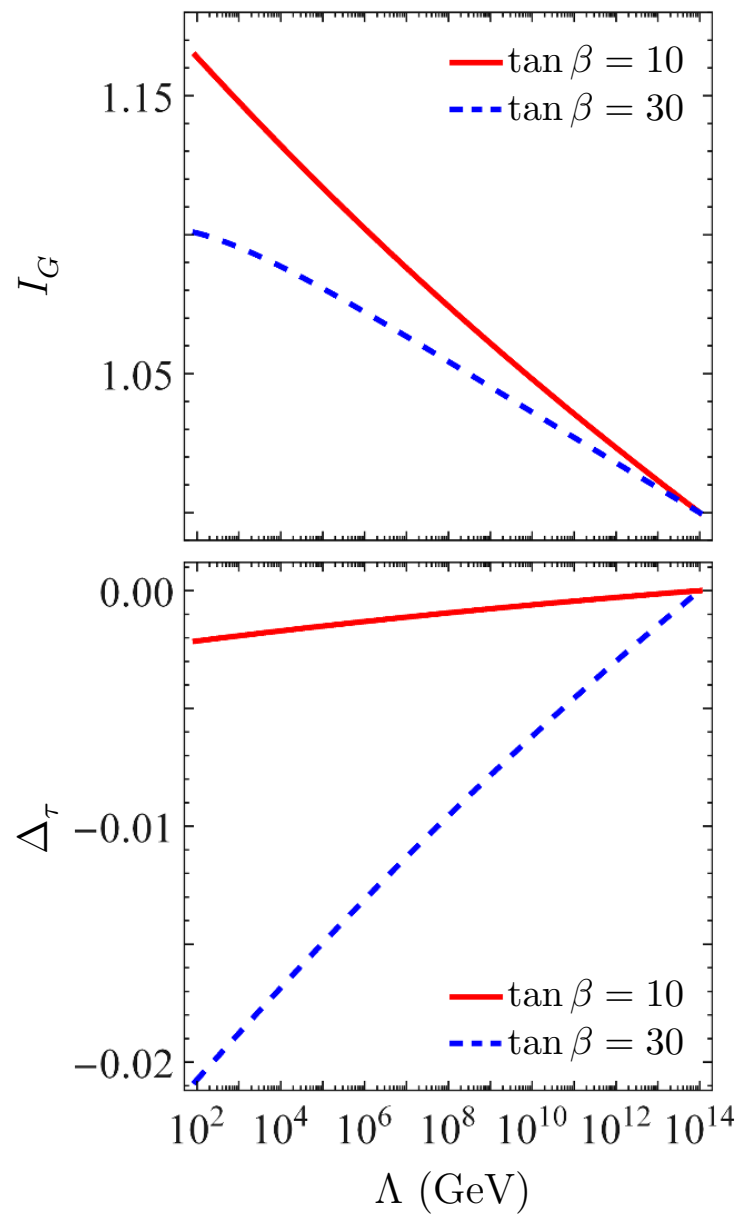

Figure 1. Changes of $I_{G}$ and $\Delta_{\tau}$ versus the energy scale $\Lambda$ in the MSSM or the type-II 2HDM.

or equivalently,

$$
M_{\nu}^{\prime} \simeq I_{G}\left[M_{\nu}-\Delta_{\tau}\left(\begin{array}{ccc}
0 & 0 & 0 \\
0 & 0 & 0 \\
e^{*} & d^{*} & c^{*}
\end{array}\right)\right],
$$

in which the smallness of $\Delta_{\tau}$ has been taken into account. It is clear that the term proportional to $\Delta_{\tau}$ measures the strength of $\mu-\tau$ symmetry breaking. Even if $M_{\nu}$ is taken to be Hermitian, the RGE-induced quantum correction will violate that Hermiticity at $\Lambda<\Lambda_{\mu \tau}$. In comparison, the Hermiticity of $H_{\nu}$ is preserved in the whole RGE evolution from $\Lambda_{\mu \tau}$ down to $\Lambda_{\mathrm{F}}$.

At this point it is worth comparing the generic expression of $H_{\nu}^{\prime}$ in eq. (2.17) with the explicit one in eq. (3.7). Of course, it is straightforward to decompose the latter into a part respecting the $\mu-\tau$ reflection symmetry and a part violating this flavor symmetry, from which one can easily obtain the dimensionless perturbation parameters

$$
\epsilon_{1} \simeq \frac{1}{2} \Delta_{\tau}, \quad \epsilon_{2} \simeq \Delta_{\tau}
$$


implying that the only source of $\mu-\tau$ reflection symmetry breaking in our example is the RGE-induced $\Delta_{\tau}$ term. In practice, it should be more convenient to directly use eq. (3.7) to do a perturbation calculation of the neutrino masses and flavor mixing parameters.

Before we start from eq. (3.7) to derive the analytical expressions of three neutrino masses and four flavor mixing parameters at $\Lambda_{\mathrm{F}}$ in the next section, let us first derive two interesting relations with no need of doing any perturbation calculation. Eq. (2.9) tells us that $H_{\nu}$ and $H_{\nu}^{\prime}$ can be diagonalized by the unitary matrices $U$ and $U^{\prime}$, respectively. So the determinants of $H_{\nu}$ and $H_{\nu}^{\prime}$ are proportional to each other, giving rise to

$$
m_{1}^{\prime} m_{2}^{\prime} m_{3}^{\prime}=I_{G}^{3} I_{e} I_{\mu} I_{\tau} m_{1} m_{2} m_{3}
$$

with $m_{i}$ and $m_{i}^{\prime}$ (for $i=1,2,3$ ) stand for the neutrino masses at $\Lambda_{\mu \tau}$ and $\Lambda_{\mathrm{F}}$, respectively. Considering the traces of $H_{\nu}$ and $H_{\nu}^{\prime}$ in eq. (3.3), we obtain

$$
\sum_{i} m_{i}^{\prime 2}=I_{G}^{2} \sum_{\alpha} I_{\alpha}^{2} \sum_{i} m_{i}^{2}\left|V_{\alpha i}\right|^{2}
$$

with $\alpha$ and $i$ running over $(e, \mu, \tau)$ and $(1,2,3)$, respectively.

But it is more interesting to establish an instructive relationship between the Jarlskog invariant of CP violation $\mathcal{J}$ at $\Lambda_{\mu \tau}$, defined through [68]

$$
\operatorname{Im}\left(V_{\alpha i} V_{\beta j} V_{\alpha j}^{*} V_{\beta i}^{*}\right)=\mathcal{J} \sum_{\gamma} \epsilon_{\alpha \beta \gamma} \sum_{k} \epsilon_{i j k}
$$

with the subscripts $(\alpha, \beta, \gamma)$ and $(i, j, k)$ running respectively over $(e, \mu, \tau)$ and $(1,2,3)$, and its counterpart $\mathcal{J}^{\prime}$ at $\Lambda_{\mathrm{F}}$. To do so, we first write out the elements of $H_{\nu}^{\prime}$ in eq. (3.3) in terms of the neutrino masses and the PMNS matrix elements:

$$
\sum_{i} m_{i}^{\prime 2} U_{\alpha i}^{\prime} U_{\beta i}^{\prime *}=I_{G}^{2} I_{\alpha} I_{\beta} \sum_{i} m_{i}^{2} U_{\alpha i} U_{\beta i}^{*}
$$

in which both $\alpha$ and $\beta$ run over $e, \mu$ and $\tau$. Note that $U=P V$ (or $U^{\prime}=P^{\prime} V^{\prime}$ ) contains three unphysical phases. To eliminate them, let us focus on the following rephasing invariant [69]:

$$
\begin{aligned}
\operatorname{Im}\left[\sum_{i} m_{i}^{2} U_{e i} U_{\mu i}^{*} \cdot \sum_{j} m_{j}^{2} U_{\mu j} U_{\tau j}^{*} \cdot \sum_{k} m_{k}^{2} U_{\tau k} U_{e k}^{*}\right] \\
=\sum_{i} \sum_{j} \sum_{k} m_{i}^{2} m_{j}^{2} m_{k}^{2} \operatorname{Im}\left(V_{e i} V_{\mu j} V_{\tau k} V_{e k}^{*} V_{\mu i}^{*} V_{\tau j}^{*}\right) \\
=\mathcal{J} \sum_{i} \sum_{j} m_{i}^{2} m_{j}^{4} \sum_{k} \epsilon_{i j k} \\
=\mathcal{J} \Delta m_{21}^{2} \Delta m_{31}^{2} \Delta m_{32}^{2}
\end{aligned}
$$

where the three neutrino mass-squared differences are defined as $\Delta m_{i j}^{2} \equiv m_{i}^{2}-m_{j}^{2}$ (for $i, j=1,2,3)$. Applying eq. (3.13) to eq. (3.14), we are then left with the elegant result

$$
\mathcal{J}^{\prime} \Delta m_{21}^{\prime 2} \Delta m_{31}^{\prime 2} \Delta m_{32}^{\prime 2}=I_{G}^{6} I_{e}^{2} I_{\mu}^{2} I_{\tau}^{2} \mathcal{J} \Delta m_{21}^{2} \Delta m_{31}^{2} \Delta m_{32}^{2},
$$


which concisely connects the strength of leptonic $\mathrm{CP}$ violation at $\Lambda_{\mu \tau}$ to that at $\Lambda_{\mathrm{F}}$. Given the parametrization of $V$ in eq. (2.11), the Jarlskog invariant $\mathcal{J}$ reads as

$$
\mathcal{J}=\frac{1}{8} \sin 2 \theta_{12} \sin 2 \theta_{13} \cos \theta_{13} \sin 2 \theta_{23} \sin \delta .
$$

If $\theta_{23}=\pi / 4$ and $\delta=\pi / 2$ or $3 \pi / 2$ are taken into account in the $\mu-\tau$ reflection symmetry limit, then we arrive at $|\mathcal{J}|=\sin 2 \theta_{12} \sin 2 \theta_{13} \cos \theta_{13} / 8$. Taking a similar parametrization for $V^{\prime}$, one may express $\mathcal{J}^{\prime}$ in terms of the corresponding flavor mixing parameters as

$$
\mathcal{J}^{\prime}=\frac{1}{8} \sin 2 \theta_{12}^{\prime} \sin 2 \theta_{13}^{\prime} \cos \theta_{13}^{\prime} \sin 2 \theta_{23}^{\prime} \sin \delta^{\prime} .
$$

In the next section we shall establish the analytical relations between $\left(\theta_{12}, \theta_{13}, \theta_{23}, \delta\right)$ at $\Lambda_{\mu \tau}$ and $\left(\theta_{12}^{\prime}, \theta_{13}^{\prime}, \theta_{23}^{\prime}, \delta^{\prime}\right)$ at $\Lambda_{\mathrm{F}}$ in a perturbation approach.

\section{RGE corrections to $U$}

Let us start from eq. (3.7) to do a perturbation calculation in order to derive the analytical expressions of three neutrino masses and four flavor mixing parameters at $\Lambda_{\mathrm{F}}$. Similar to $H_{\nu}$ in eq. (2.9), $H_{\nu}^{\prime}$ can also be reconstructed in the same way:

$$
H_{\nu}^{\prime} \equiv M_{\nu}^{\prime} M_{\nu}^{\prime \dagger}=U^{\prime} \hat{M}_{\nu}^{\prime 2} U^{\prime \dagger},
$$

in which $U^{\prime}=P^{\prime} V^{\prime}$ with $P^{\prime}$ being a diagonal phase matrix, and $\hat{M}_{\nu}^{\prime} \equiv \operatorname{Diag}\left\{m_{1}^{\prime}, m_{2}^{\prime}, m_{3}^{\prime}\right\}$ with $m_{i}^{\prime}$ being the neutrino masses at $\Lambda_{\mathrm{F}}$. Then the approximate relationship between $H_{\nu}^{\prime}$ and $H_{\nu}$ in eq. (3.7) can be rewritten as

$$
\hat{M}_{\nu}^{\prime 2} \simeq I_{G}^{2} U^{\prime \dagger}\left[U \hat{M}_{\nu}^{2} U^{\dagger}-\Delta_{\tau}\left(\begin{array}{ccc}
0 & 0 & \sum_{i} m_{i}^{2} U_{e i} U_{\tau i}^{*} \\
0 & 0 & \sum_{i} m_{i}^{2} U_{\mu i} U_{\tau i}^{*} \\
\sum_{i} m_{i}^{2} U_{e i}^{*} U_{\tau i} & \sum_{i} m_{i}^{2} U_{\mu i}^{*} U_{\tau i} & 2 \sum_{i} m_{i}^{2}\left|U_{\tau i}\right|^{2}
\end{array}\right)\right] U^{\prime} .
$$

Treating $\Delta_{\tau}$ as a small perturbation parameter, let us define the RGE-induced deviations of the relevant flavor mixing angles and phase parameters at $\Lambda_{\mathrm{F}}$ from their original counterparts at $\Lambda_{\mu \tau}$ as follows:

$$
\begin{aligned}
\Delta \theta_{12} & =\theta_{12}^{\prime}-\theta_{12}, & \Delta \delta & =\delta^{\prime}-\delta, \\
\Delta \theta_{13} & =\theta_{13}^{\prime}-\theta_{13}, & \Delta \phi_{e \mu} & =\left(\phi_{e}^{\prime}-\phi_{\mu}^{\prime}\right)-\left(\phi_{e}-\phi_{\mu}\right), \\
\Delta \theta_{23} & =\theta_{23}^{\prime}-\theta_{23}, & \Delta \phi_{e \tau} & =\left(\phi_{e}^{\prime}-\phi_{\tau}^{\prime}\right)-\left(\phi_{e}-\phi_{\tau}\right),
\end{aligned}
$$

which are expected to be small enough in magnitude as compared with their respective starting values at $\Lambda_{\mu \tau}$. Note that $\theta_{23}=\pi / 4$ and $\delta=\pi / 2$ or $3 \pi / 2$ at the $\mu-\tau$ reflection symmetry scale $\Lambda_{\mu \tau}$ will be implied in the subsequent perturbation calculations. Note also that only two combinations of the three unphysical phases in $P$ or $P^{\prime}$, as indicated in eq. (4.3), are associated with our derivation of the RGEs for the physical parameters. They ought not to be ignored in the course of the calculations, but of course they do not show 
up in the final results of $\Delta \theta_{12}, \Delta \theta_{13}, \Delta \theta_{23}$ and $\Delta \delta$. Next we expand the elements of $\hat{M}_{\nu}^{\prime 2}$ in terms of the above perturbation parameters and only keep their first-order contributions.

First of all, it is straightforward to obtain the analytical results of three neutrino masses from the diagonal elements of $\hat{M}_{\nu}^{\prime 2}$. Namely,

$$
\begin{aligned}
& m_{1}^{\prime} \simeq I_{G} m_{1}\left[1-\frac{1}{2} \Delta_{\tau}\left(s_{12}^{2} c_{13}^{2}+s_{13}^{2}\right)\right], \\
& m_{2}^{\prime} \simeq I_{G} m_{2}\left[1-\frac{1}{2} \Delta_{\tau}\left(c_{12}^{2} c_{13}^{2}+s_{13}^{2}\right)\right], \\
& m_{3}^{\prime} \simeq I_{G} m_{3}\left[1-\frac{1}{2} \Delta_{\tau} c_{13}^{2}\right] .
\end{aligned}
$$

Obviously but interestingly, $m_{i}^{\prime} / m_{i} \simeq I_{G}$ holds in the leading-order approximation, implying that the three neutrino masses almost run in step. Given $I_{e} \simeq I_{\mu} \simeq 1$ and $I_{\tau} \simeq 1-\Delta_{\tau}$ and the $\mu-\tau$ reflection symmetry at $\Lambda_{\mu \tau}$, it is easy to check that the product of $m_{1}^{\prime}, m_{2}^{\prime}$ and $m_{3}^{\prime}$ in eq. (4.4) can successfully reproduce the elegant relationship achieved in eq. (3.10). Moreover, eq. (4.4) leads us to the sum rule

$$
\sum_{i} m_{i}^{\prime 2} \simeq I_{G}^{2} \sum_{i} m_{i}^{2}\left(1-2 \Delta_{\tau}\left|V_{\tau i}\right|^{2}\right)
$$

which is consistent with the more generic one derived in eq. (3.11) if the same approximations are made and the $\mu-\tau$ reflection symmetry is taken into account.

Second, the off-diagonal elements of $\hat{M}_{\nu}^{\prime 2}$ in eq. (4.2) must vanish, yielding the following six constraint equations in our analytical approximations:

$$
\begin{aligned}
& 2 \Delta m_{21}^{2} \Delta \theta_{12}+\eta s_{13} \Delta m_{21}^{2}\left(\Delta \phi_{e \mu}-\Delta \phi_{e \tau}\right)-c_{12} s_{12} c_{13}^{2} \bar{m}_{12} \Delta_{\tau} \simeq 0, \\
& 2\left(c_{12}^{2}-s_{12}^{2}\right) s_{13} \Delta m_{21}^{2} \Delta \theta_{23}-\eta c_{12} s_{12} \Delta m_{21}^{2}\left[2 s_{13}^{2} \Delta \delta+c_{13}^{2}\left(\Delta \phi_{e \mu}+\Delta \phi_{e \tau}\right)\right]+s_{13} \bar{m}_{12} \Delta_{\tau} \simeq 0, \\
& 2 s_{12} c_{13} \Delta m_{31}^{2} \Delta \theta_{23}+\eta c_{12} c_{13} s_{13} \Delta m_{31}^{2}\left(2 \Delta \delta-\Delta \phi_{e \mu}-\Delta \phi_{e \tau}\right)-s_{12} c_{13} \bar{m}_{13} \Delta_{\tau} \simeq 0 \\
& 2 c_{12} \Delta m_{31}^{2} \Delta \theta_{13}-\eta s_{12} c_{13} \Delta m_{31}^{2}\left(\Delta \phi_{e \mu}-\Delta \phi_{e \tau}\right)-c_{12} c_{13} s_{13} \bar{m}_{13} \Delta_{\tau} \simeq 0, \\
& 2 c_{12} c_{13} \Delta m_{32}^{2} \Delta \theta_{23}-\eta s_{12} c_{13} s_{13} \Delta m_{32}^{2}\left(2 \Delta \delta-\Delta \phi_{e \mu}-\Delta \phi_{e \tau}\right)-c_{12} c_{13} \bar{m}_{23} \Delta_{\tau} \simeq 0, \\
& 2 s_{12} \Delta m_{32}^{2} \Delta \theta_{13}+\eta c_{12} c_{13} \Delta m_{32}^{2}\left(\Delta \phi_{e \mu}-\Delta \phi_{e \tau}\right)-s_{12} c_{13} s_{13} \bar{m}_{23} \Delta_{\tau} \simeq 0,
\end{aligned}
$$

where $\eta \equiv \sin \delta= \pm 1$ in the $\mu-\tau$ reflection symmetry limit, and $\bar{m}_{i j} \equiv m_{i}^{2}+m_{j}^{2}$ (for $i, j=1,2,3)$. Solving the above equations, we obtain

$$
\begin{aligned}
& \Delta \theta_{12} \simeq \Delta_{\tau} s_{12} c_{12}\left[\frac{m_{1}^{2}+m_{2}^{2}}{2 \Delta m_{21}^{2}} c_{13}^{2}-\frac{m_{3}^{2} \Delta m_{21}^{2}}{\Delta m_{31}^{2} \Delta m_{32}^{2}} s_{13}^{2}\right], \\
& \Delta \theta_{13} \simeq \Delta_{\tau} s_{13} c_{13}\left[\frac{m_{2}^{2}+m_{3}^{2}}{2 \Delta m_{32}^{2}} s_{12}^{2}+\frac{m_{1}^{2}+m_{3}^{2}}{2 \Delta m_{31}^{2}} c_{12}^{2}\right], \\
& \Delta \theta_{23} \simeq \Delta_{\tau}\left[\frac{m_{2}^{2}+m_{3}^{2}}{2 \Delta m_{32}^{2}} c_{12}^{2}+\frac{m_{1}^{2}+m_{3}^{2}}{2 \Delta m_{31}^{2}} s_{12}^{2}\right]
\end{aligned}
$$


and

$$
\begin{aligned}
\Delta \delta & \simeq \eta \Delta_{\tau}\left[\frac{m_{1}^{2} \Delta m_{32}^{2}}{\Delta m_{21}^{2} \Delta m_{31}^{2}} t_{12} s_{13}+\frac{m_{2}^{2} \Delta m_{31}^{2}}{\Delta m_{21}^{2} \Delta m_{32}^{2}} \cdot \frac{s_{13}}{t_{12}}-\frac{m_{3}^{2} \Delta m_{21}^{2}}{\Delta m_{31}^{2} \Delta m_{32}^{2}} s_{12} c_{12}\left(\frac{1}{s_{13}}+s_{13}\right)\right], \\
\Delta \phi_{e \mu} & \simeq \eta \Delta_{\tau} s_{13}\left[\frac{m_{1}^{2} \Delta m_{32}^{2}}{\Delta m_{21}^{2} \Delta m_{31}^{2}} t_{12}+\frac{m_{2}^{2} \Delta m_{31}^{2}}{\Delta m_{21}^{2} \Delta m_{32}^{2}} \cdot \frac{1}{t_{12}}\right], \\
\Delta \phi_{e \tau} & \simeq \eta \Delta_{\tau} s_{13}\left[\frac{m_{1}^{2} \Delta m_{32}^{2}}{\Delta m_{21}^{2} \Delta m_{31}^{2}} t_{12}+\frac{m_{2}^{2} \Delta m_{31}^{2}}{\Delta m_{21}^{2} \Delta m_{32}^{2}} \cdot \frac{1}{t_{12}}-\frac{2 m_{3}^{2} \Delta m_{21}^{2}}{\Delta m_{31}^{2} \Delta m_{32}^{2}} s_{12} c_{12}\right],
\end{aligned}
$$

where $t_{12} \equiv \tan \theta_{12}$. One can see that the RGE-induced corrections to all the four flavor mixing parameters are proportional to $\Delta_{\tau}$, a fact which is under rational expectation. Among the three angles, $\theta_{12}$ is more sensitive to the quantum corrections than $\theta_{13}$ and $\theta_{23}$ in most cases, mainly because of $\left|\Delta m_{31}^{2}\right| \simeq\left|\Delta m_{32}^{2}\right| \sim 30 \Delta m_{21}^{2}$ [70, 71]. On the other hand, the smallness of $s_{13}$ [72] implies that the magnitude of $\Delta \theta_{13}$ must be smaller than that of $\Delta \theta_{23}$. But the expression of $\Delta \delta$ contains three terms proportional to $s_{13}$ and one term proportional to $1 / s_{13}$, and hence the overall running effect of $\delta$ is generally expected to be more significant than those of three flavor mixing angles, or at least than those of $\theta_{13}$ and $\theta_{23}$. Note that $\phi_{\mu}+\phi_{\tau}=2 \phi_{e}$ holds at $\Lambda_{\mu \tau}$ due to the $\mu$ - $\tau$ reflection symmetry of $H_{\nu}$, and hence $2 \phi_{e}^{\prime}-\phi_{\mu}^{\prime}-\phi_{\tau}^{\prime}=\Delta \phi_{e \mu}+\Delta \phi_{e \tau} \propto \Delta_{\tau}$ is not vanishing at $\Lambda_{\mathrm{F}}$, providing us with another (unphysical) measure of the RGE-induced $\mu-\tau$ reflection symmetry breaking of $H_{\nu}^{\prime}$.

There are two ways to calculate the Jarlskog invariant $\mathcal{J}^{\prime}$ at $\Lambda_{\mathrm{F}}$ : one is to apply eq. (4.4) to the elegant relationship between $\mathcal{J}$ and $\mathcal{J}^{\prime}$ in eq. (3.15) with $I_{e} \simeq I_{\mu} \simeq 1$ and $I_{\tau} \simeq 1-\Delta_{\tau}$, and the other is to do a direct perturbation calculation of $\mathcal{J}^{\prime}$ by using eqs. (3.17), (4.7) and (4.8). After doing such a calculation, we obtain the ratio of $\mathcal{J}^{\prime}$ at $\Lambda_{\mathrm{F}}$ to $\mathcal{J}$ at $\Lambda_{\mu \tau}$ as follows:

$$
\frac{\mathcal{J}^{\prime}}{\mathcal{J}} \simeq 1+\Delta_{\tau}\left[\left(s_{12}^{2} c_{13}^{2}-s_{13}^{2}\right)\left(\frac{m_{2}^{2}}{\Delta m_{32}^{2}}-\frac{m_{1}^{2}}{\Delta m_{21}^{2}}\right)+\left(c_{12}^{2} c_{13}^{2}-s_{13}^{2}\right)\left(\frac{m_{1}^{2}}{\Delta m_{31}^{2}}+\frac{m_{2}^{2}}{\Delta m_{21}^{2}}\right)\right] .
$$

Different from $\delta, \mathcal{J}$ evolves in a way insensitive to the smallness of $\theta_{13}$.

We proceed to numerically illustrate the RGE-induced corrections to the neutrino masses and flavor mixing parameters in the MSSM and the type-II 2HDM by using the program advocated in ref. [73] and taking $\Lambda_{\mu \tau}=10^{14} \mathrm{GeV}$ as a typical choice, where $\theta_{23}=\pi / 4$ and $\delta=3 \pi / 2$ are input. For the sake of simplicity, we adjust the initial values of $m_{1}$ (or $\left.m_{3}\right), \Delta m_{21}^{2}, \Delta m_{31}^{2}, \theta_{12}$ and $\theta_{13}$ to make sure that all the neutrino oscillation parameters can be compatible with current experimental data at $\Lambda_{\mathrm{F}}[70,71]$. The main numerical results are summarized in tables 1 and 2 as well as figures 2, 3 and 4, in which two possibilities of the neutrino mass spectrum have been taken into account - the normal hierarchy (NH) with $m_{1}<m_{2}<m_{3}$ or $\Delta m_{31}^{2}>0$ and the inverted hierarchy (IH) with $m_{3}<m_{1}<m_{2}$ or $\Delta m_{31}^{2}<0$. Some comments and discussions are in order.

(1) In the MSSM, table 1 and figure 2 show that the values of three flavor mixing angles increase in the $\mathrm{NH}$ case as the energy scale $\Lambda$ decreases, but $\theta_{13}$ and $\theta_{23}$ decrease in the IH case as $\Lambda$ decreases. In either case a larger value of $\tan \beta$ will enhance the running effects. Such a direction of evolution of $\Delta \theta_{i j}$ (for $i j=12,13$ or 23 ) can easily be understood from 
MSSM
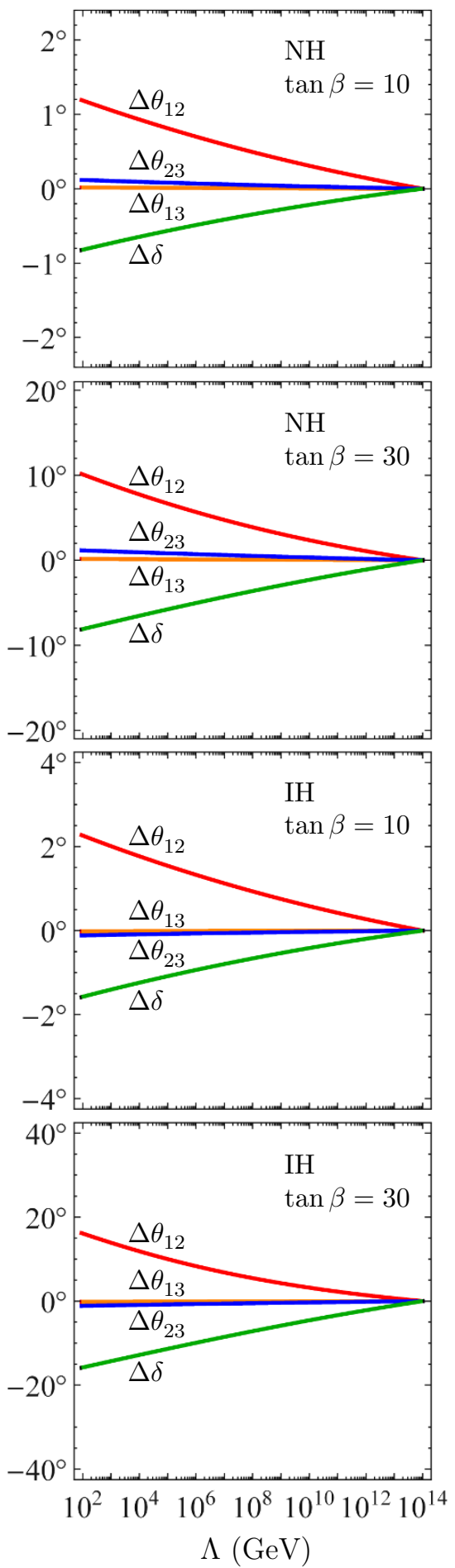

$2 \mathrm{HDM}$
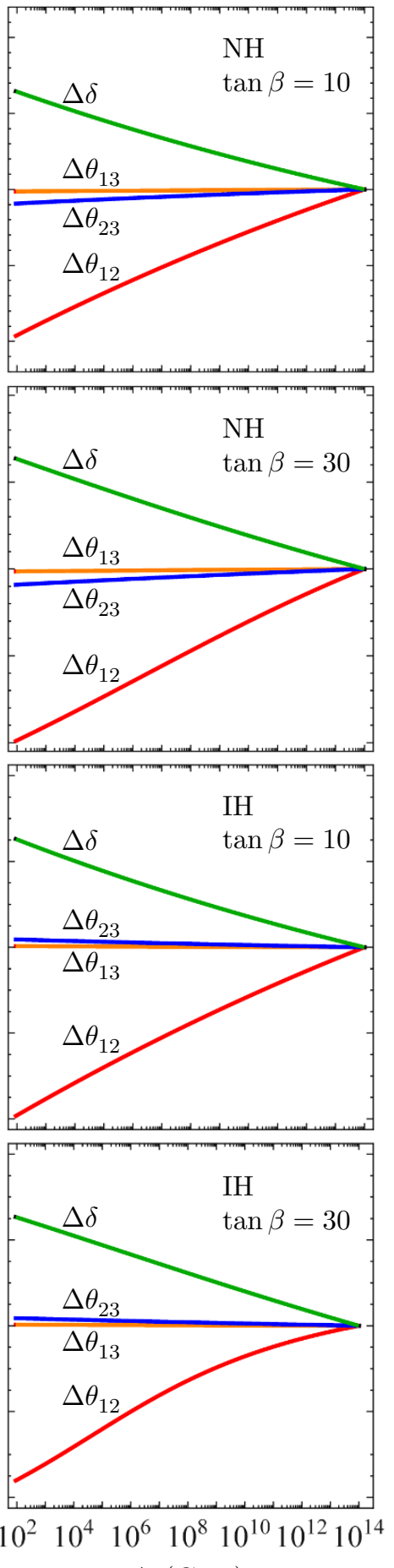

Figure 2. The changes of $\Delta \theta_{12}, \Delta \theta_{13}, \Delta \theta_{23}$ and $\Delta \delta$ with the energy scale $\Lambda$ in the MSSM and the type-II $2 \mathrm{HDM}$, where $m_{\text {lightest }}=0.05 \mathrm{eV}$ at $\Lambda_{\mathrm{F}}=10^{2} \mathrm{GeV}$ is typically input and $\theta_{23}=\pi / 4$ and $\delta=3 \pi / 2$ at $\Lambda_{\mu \tau}=10^{14} \mathrm{GeV}$ are fixed by the $\mu-\tau$ reflection symmetry. 


\begin{tabular}{|lllllllll|}
\hline MSSM & \multicolumn{2}{c}{$\mathrm{NH}, \tan \beta=10$} & $\mathrm{NH}, \tan \beta=30$ & \multicolumn{2}{c|}{$\mathrm{IH}, \tan \beta=10$} & \multicolumn{2}{c|}{$\mathrm{IH}, \tan \beta=30$} \\
Parameter & $\Lambda_{\mu \tau}$ & $\Lambda_{\mathrm{F}}$ & $\Lambda_{\mu \tau}$ & $\Lambda_{\mathrm{F}}$ & $\Lambda_{\mu \tau}$ & $\Lambda_{\mathrm{F}}$ & $\Lambda_{\mu \tau}$ & $\Lambda_{\mathrm{F}}$ \\
\hline$m_{\text {lightest }}\left[10^{-2} \mathrm{eV}\right]$ & 5.4 & 5.06 & 5.4 & 5.03 & 5.36 & 5.02 & 5.4 & 5.00 \\
$\Delta m_{21}^{2}\left[10^{-5} \mathrm{eV}^{2}\right]$ & 8.77 & 7.56 & 10.77 & 7.56 & 8.92 & 7.56 & 13.19 & 7.56 \\
$\left|\Delta m_{31}^{2}\right|\left[10^{-3} \mathrm{eV}^{2}\right]$ & 2.91 & 2.55 & 3.00 & 2.55 & 2.84 & 2.49 & 2.84 & 2.49 \\
$\theta_{12}\left[^{\circ}\right]$ & 33.31 & 34.50 & 24.36 & 34.51 & 32.23 & 34.50 & 18.30 & 34.49 \\
$\theta_{13}\left[^{\circ}\right]$ & 8.42 & 8.44 & 8.28 & 8.44 & 8.43 & 8.41 & 8.58 & 8.41 \\
$\theta_{23}\left[^{\circ}\right]$ & 45 & 45.12 & 45 & 46.17 & 45 & 44.89 & 45 & 43.87 \\
$\delta\left[^{\circ}\right]$ & 270 & 269.18 & 270 & 261.87 & 270 & 268.42 & 270 & 254.15 \\
\hline $\mathcal{J}\left[^{10-2}\right]$ & -3.29 & -3.35 & -2.65 & -3.32 & -3.24 & -3.34 & -2.17 & -3.21 \\
\hline
\end{tabular}

Table 1. An illustration of the neutrino oscillation parameters at $\Lambda_{\mu \tau}$ and $\Lambda_{\mathrm{F}}$ in the MSSM with $\tan \beta=10$ or 30 , where both $\mathrm{NH}$ and $\mathrm{IH}$ cases are considered.

\begin{tabular}{|lllllllll|}
\hline $2 \mathrm{HDM}$ & \multicolumn{2}{c}{$\mathrm{NH}, \tan \beta=10$} & $\mathrm{NH}, \tan \beta=30$ & \multicolumn{2}{c|}{$\mathrm{IH}, \tan \beta=10$} & \multicolumn{2}{c|}{$\mathrm{IH}, \tan \beta=30$} \\
Parameter & $\Lambda_{\mu \tau}$ & $\Lambda_{\mathrm{F}}$ & $\Lambda_{\mu \tau}$ & $\Lambda_{\mathrm{F}}$ & $\Lambda_{\mu \tau}$ & $\Lambda_{\mathrm{F}}$ & $\Lambda_{\mu \tau}$ & $\Lambda_{\mathrm{F}}$ \\
\hline$m_{\text {lightest }}\left[10^{-2} \mathrm{eV}\right]$ & 4.29 & 5.0 & 4.53 & 5.01 & 4.29 & 5.0 & 4.5 & 5.0 \\
$\Delta m_{21}^{2}\left[10^{-5} \mathrm{eV}^{2}\right]$ & 5.44 & 7.56 & 5.94 & 7.56 & 5.34 & 7.56 & 8.31 & 7.56 \\
$\left|\Delta m_{31}^{2}\right|\left[10^{-3} \mathrm{eV}^{2}\right]$ & 1.88 & 2.55 & 2.04 & 2.55 & 1.84 & 2.49 & 2.04 & 2.49 \\
$\theta_{12}\left[^{\circ}\right]$ & 36.43 & 34.50 & 54.36 & 34.55 & 38.45 & 34.52 & 70.6 & 34.52 \\
$\theta_{13}\left[^{\circ}\right]$ & 8.47 & 8.44 & 8.72 & 8.44 & 8.38 & 8.41 & 8.17 & 8.41 \\
$\theta_{23}\left[^{\circ}\right]$ & 45 & 44.81 & 45 & 43.18 & 45 & 45.18 & 45 & 46.77 \\
$\delta\left[^{\circ}\right]$ & 270 & 271.29 & 270 & 282.71 & 270 & 272.52 & 270 & 295.34 \\
\hline $\mathcal{J}\left[1^{-2}\right]$ & -3.44 & -3.35 & -3.51 & -3.27 & -3.47 & -3.34 & -2.18 & -3.01 \\
\hline
\end{tabular}

Table 2. An illustration of the neutrino oscillation parameters at $\Lambda_{\mu \tau}$ and $\Lambda_{\mathrm{F}}$ in the type-II $2 \mathrm{HDM}$ with $\tan \beta=10$ or 30 , where both $\mathrm{NH}$ and $\mathrm{IH}$ cases are considered.

our analytical approximations made in eq. (4.7). In comparison, the CP-violating phase $\delta$ decreases in both $\mathrm{NH}$ and $\mathrm{IH}$ cases when $\Lambda$ becomes lower. The reason for this behavior can be seen in eq. (4.8) - namely, $\delta=3 \pi / 2$ (or $\eta=-1$ ) has been input at $\Lambda_{\mu \tau}$, and $\Delta \delta$ is essentially insensitive to the sign of $\Delta m_{31}^{2}$ which is always the same as the sign of $\Delta m_{32}^{2}$. Moreover, both table 1 and figure 3 tell us that the magnitude of the Jarlskog invariant (i.e., $|\mathcal{J}|)$ increases as $\Lambda$ decreases, no matter whether the neutrino mass hierarchy is normal or inverted. Eq. (4.9) shows that the ratio $\mathcal{J}^{\prime} / \mathcal{J}$ must be slightly larger than one if the term proportional to $m_{2}^{2} / \Delta m_{21}^{2}$ is dominant. Although the above observations are more or less subject to the limited parameter space that we have taken into account, our analytical results in eqs. (4.7), (4.8) and (4.9) are certainly more general and more useful. 
MSSM
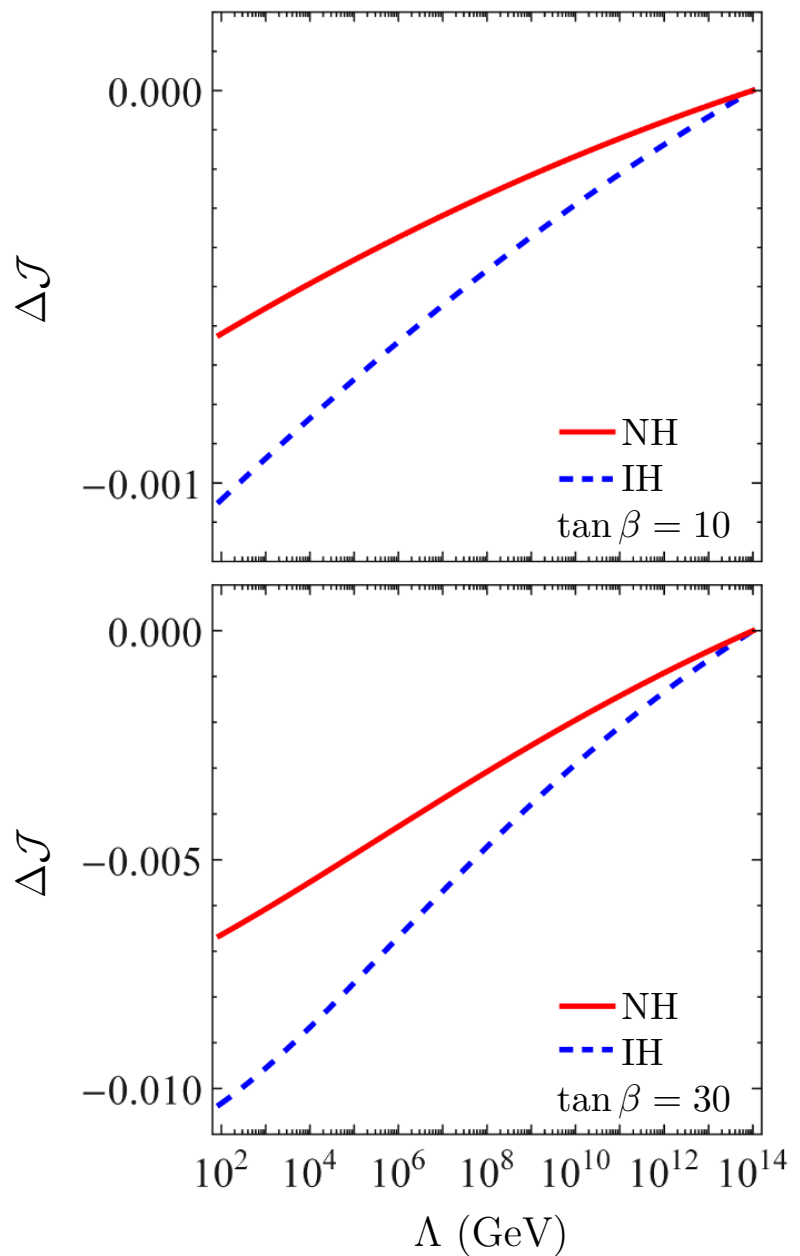

$2 \mathrm{HDM}$
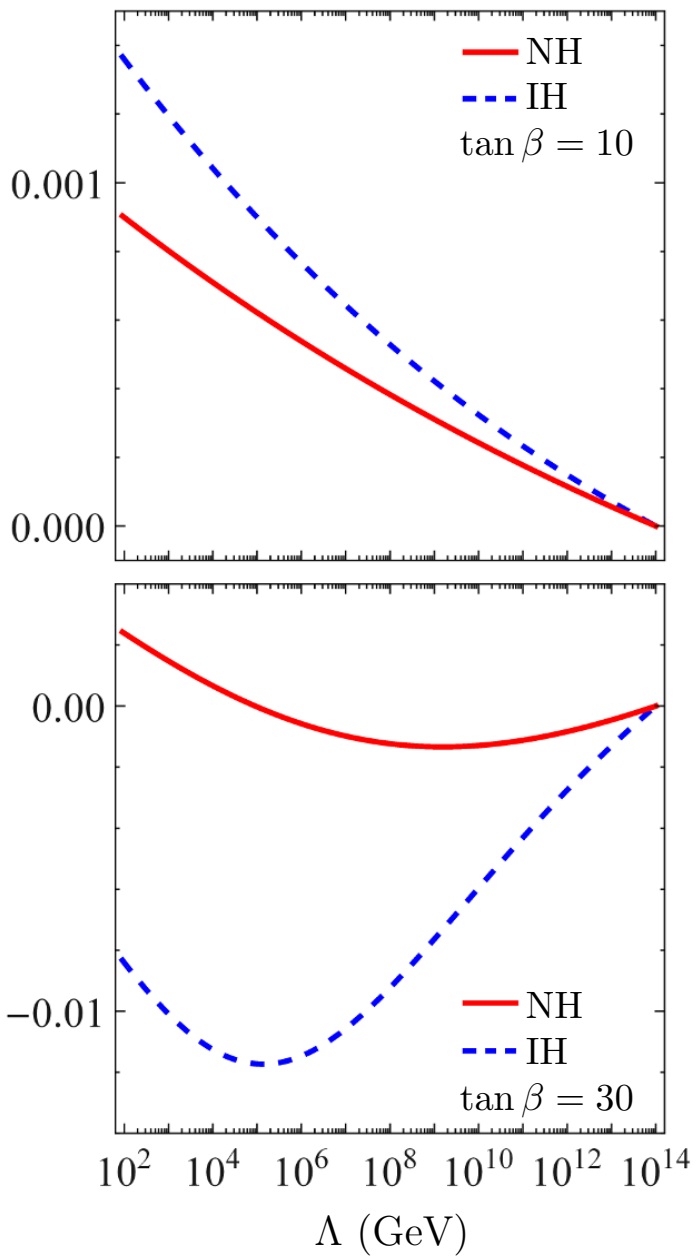

Figure 3. An illustration of the change of $\Delta \mathcal{J} \equiv \mathcal{J}^{\prime}-\mathcal{J}$ with the energy scale $\Lambda$ in the MSSM and the type-II $2 \mathrm{HDM}$, where $m_{\text {lightest }}=0.05 \mathrm{eV}$ at $\Lambda_{\mathrm{F}}=10^{2} \mathrm{GeV}$ is typically input and $\theta_{23}=\pi / 4$ and $\delta=3 \pi / 2$ at $\Lambda_{\mu \tau}=10^{14} \mathrm{GeV}$ are fixed by the $\mu-\tau$ reflection symmetry.

(2) In the type-II $2 \mathrm{HDM}$, the running behaviors of $\theta_{12}, \theta_{13}, \theta_{23}$ and $\delta$ take the opposite directions as compared with those in the MSSM. The reason is simply that the signs of $\Delta_{\tau}$ are opposite in these two scenarios. Because of $C_{l}=1$ in the MSSM and $C_{l}=-3 / 2$ in the type-II $2 \mathrm{HDM}$, the magnitude $\Delta_{\tau}$ in the latter case is about 1.5 times larger than that in the former case. That is why we have taken the type-II 2HDM scenario for our numerical illustration, in contrast with the MSSM scenario. Note, however, that the evolution of $\Delta \mathcal{J}$ with $\Lambda$ is a bit subtle in the type-II $2 \mathrm{HDM}$ case when $\tan \beta$ is sufficiently large. For example, the minimum of $\Delta \mathcal{J}$ shown in the right-bottom panel of figure 3 is expected to arise from a significant cancellation among the terms on the right-hand side of eq. (4.9).

(3) It is worth highlighting that the RGE-induced effect of $\mu-\tau$ reflection symmetry breaking provides a model-independent way to connect three burning issues in today's neutrino physics: the neutrino mass ordering, the octant of $\theta_{23}$ and leptonic CP violation. Some in- 
teresting works have been done in this regard in the case that the massive neutrinos are the Majorana particles [51, 52, 54-57, 74, 75]. Here we have discussed how the $\mu$ - $\tau$ reflection symmetry of Dirac neutrinos can be spontaneously broken by the RGE evolution from $\Lambda_{\mu \tau}$ down to $\Lambda_{\mathrm{F}}$ in the MSSM and the type-II $2 \mathrm{HDM}$, and how this symmetry breaking affects the octant of $\theta_{23}$ and the quadrant of $\delta$ in both $\mathrm{NH}$ and IH cases. As shown in figure 2, the type-II $2 \mathrm{HDM}$ scenario seems to be somewhat favored if we stick to the best-fit value of $\theta_{23}$ at low energy scales [70,71], which lies in the first octant in the NH case but in the second octant in the IH case. ${ }^{11}$ For the time being, however, the "best-fit" values of $\theta_{23}$ from a global analysis of current neutrino oscillation data should not be taken too seriously, because their statistical significance remains rather poor [70, 71]. It is more appropriate to consider the $2 \sigma$ or $3 \sigma$ intervals of those neutrino oscillation parameters, in which case the octant of $\theta_{23}$ is not yet fixed. ${ }^{12}$

(4) As a by-product, figure 4 illustrates the evolution behaviors of three neutrino masses in both NH and IH cases. Since we have intended to take $m_{\text {lightest }}=0.05 \mathrm{eV}$ at $\Lambda_{\mathrm{F}}$ in our numerical calculations so as to reasonably magnify the RGE running effects, the neutrino mass spectrum is not far away from the nearly degenerate case with a fine split between $m_{1}$ and $m_{2}$ even if it is normal. Our numerical results are consistent with the analytical ones obtained in eq. (4.4) - namely, the evolution of $m_{i}$ is mainly governed by that of $I_{G}$ and thus insensitive to the value of $\tan \beta$. For the same reason, the results of $m_{i}$ in the MSSM are not very different from those in the type-II 2HDM.

\section{Summary}

While the nature of massive neutrinos (i.e., whether Dirac or Majorana) remains an intriguing puzzle in particle physics, it is largely believed that there should exist an approximate $\mu-\tau$ reflection symmetry behind the observed pattern of lepton flavor mixing. In this work we have studied such a simple but interesting flavor symmetry for the Dirac neutrino mass matrix, which can naturally predict $\theta_{23}=\pi / 4$ and $\delta=\pi / 2$ or $3 \pi / 2$ in the standard parametrization of the PMNS matrix $U$. Assuming the $\mu-\tau$ reflection symmetry is realized at a superhigh energy scale $\Lambda_{\mu \tau}$, we have investigated how it is spontaneously broken via the one-loop RGEs running from $\Lambda_{\mu \tau}$ down to the Fermi scale $\Lambda_{\mathrm{F}}$ in two interesting scenarios: the MSSM and the type-II 2HDM. Such quantum corrections to the neutrino masses and flavor mixing parameters have been derived in a perturbation approach, and an analytical link has also been established between the Jarlskog invariants of leptonic CP violation at $\Lambda_{\mu \tau}$ and $\Lambda_{\mathrm{F}}$. In addition, we have illustrated the running behaviors of relevant physical quantities by taking a few typical numerical examples in the MSSM and the type-II 2HDM.

\footnotetext{
${ }^{11}$ If one works on the RGEs in the SM framework, then $\Delta \theta_{i j}$ and $\Delta \delta$ will evolve with the energy scales in a similar way as in the type-II 2 HDM scenario. In this case, however, the running effects of relevant parameters are expected to be much milder because of the lack of the $\tan \beta$ enhancement. More seriously, the SM-like RGEs may suffer from the vacuum-stability problem as the energy scale is above $10^{10} \mathrm{GeV}[66,67]$.

${ }^{12} \mathrm{At}$ this point it is worth mentioning that the latest $\mathrm{T} 2 \mathrm{~K}$ neutrino oscillation result provides a very preliminary hint that $\theta_{23}$ might lie in the second octant in the $\mathrm{NH}$ case [76, 77], a possibility compatible with our results in the MSSM scenario shown in table 1 and figure 2.
} 


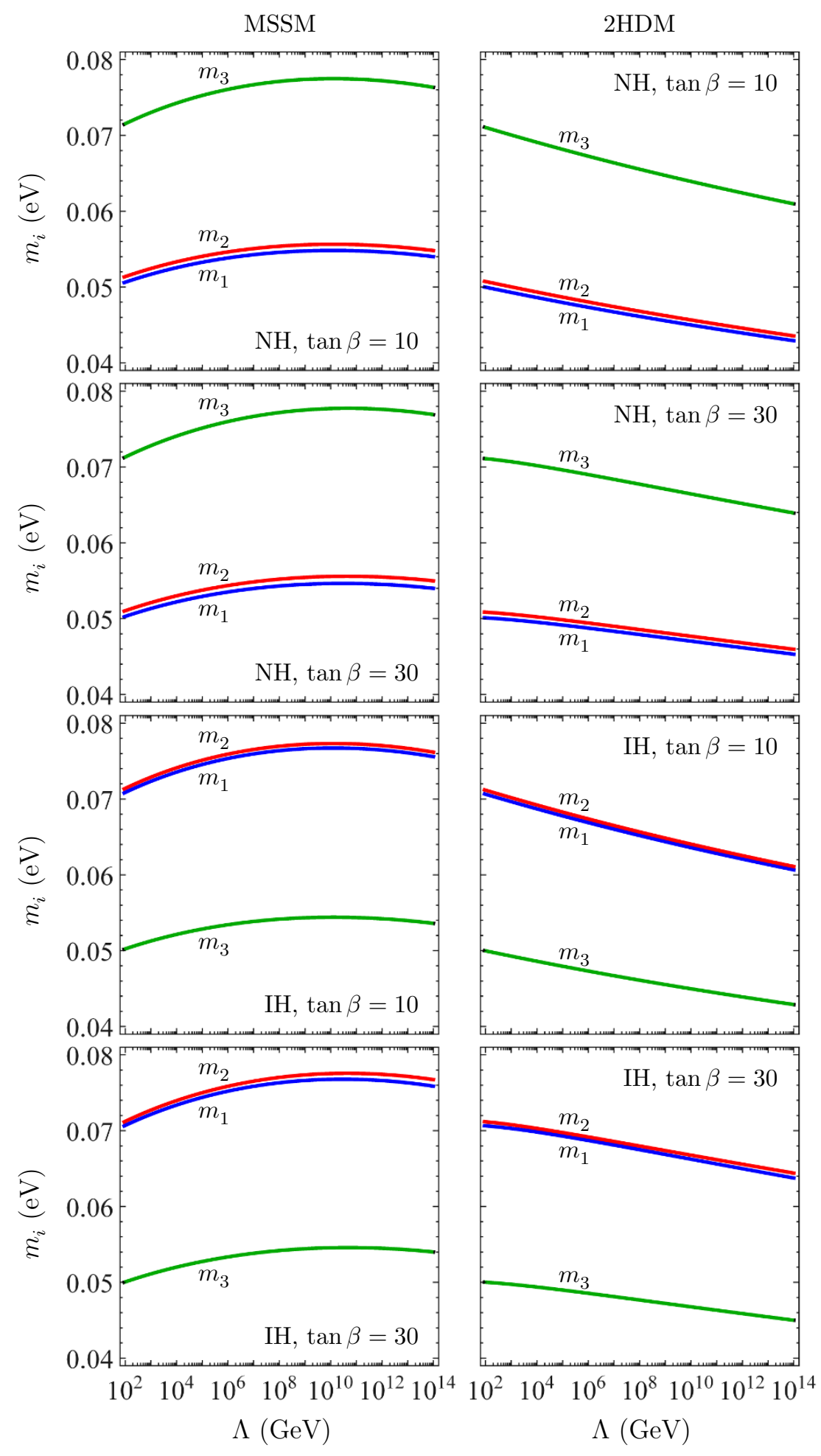

Figure 4. The three neutrino masses evolving with the energy scale $\Lambda$ in the MSSM and the type-II $2 \mathrm{HDM}$, where $m_{\text {lightest }}=0.05 \mathrm{eV}$ at $\Lambda_{\mathrm{F}}=10^{2} \mathrm{GeV}$ is typically input and $\theta_{23}=\pi / 4$ and $\delta=3 \pi / 2$ at $\Lambda_{\mu \tau}=10^{14} \mathrm{GeV}$ are fixed by the $\mu-\tau$ reflection symmetry. 
A particularly striking point of view associated with this kind of study is that the octant of $\theta_{23}$, the quadrant of $\delta$ and the neutrino mass ordering might be correlated with one another thanks to the RGE-triggered breaking of $\mu-\tau$ reflection symmetry. We have illustrated this observation both analytically and numerically by considering the massive Dirac neutrinos in the MSSM and the type-II 2HDM, and found that these two scenarios lead us to the opposite deviations of $\theta_{12}, \theta_{13}, \theta_{23}$ and $\delta$ from their corresponding values in the $\mu$ - $\tau$ reflection symmetry limit. Therefore, the future experimental data on the neutrino mass ordering and flavor mixing angles will allow us to make a choice between the MSSM and the type-II 2HDM, at least in this connection. Our results are also expected to be useful for building explicit Dirac neutrino mass models and explaining upcoming neutrino oscillation data.

\section{Acknowledgments}

We would like to thank S. Antusch, G.Y. Huang, S. Luo, J. Zhang, Z.H. Zhao and S. Zhou for useful discussions. This research work was supported in part by the National Natural Science Foundation of China under grant No. 11375207 and grant No. 11775231.

Open Access. This article is distributed under the terms of the Creative Commons Attribution License (CC-BY 4.0), which permits any use, distribution and reproduction in any medium, provided the original author(s) and source are credited.

\section{References}

[1] Particle Data Group collaboration, C. Patrignani et al., Review of particle physics, Chin. Phys. C 40 (2016) 100001 [inSPIRE].

[2] J.-W. Mei and Z.-Z. Xing, Radiative corrections to neutrino mixing and CP-violation in the minimal seesaw model with leptogenesis, Phys. Rev. D 69 (2004) 073003 [hep-ph/0312167] [INSPIRE].

[3] G.C. Branco, P.M. Ferreira, L. Lavoura, M.N. Rebelo, M. Sher and J.P. Silva, Theory and phenomenology of two-Higgs-doublet models, Phys. Rept. 516 (2012) 1 [arXiv:1106. 0034] [INSPIRE].

[4] E. Majorana, Teoria simmetrica dell'elettrone e del positrone (in Italian), Nuovo Cim. 14 (1937) 171 [INSPIRE].

[5] P. Minkowski, $\mu \rightarrow e \gamma$ at a rate of one out of $10^{9}$ muon decays?, Phys. Lett. B 67 (1977) 421 [INSPIRE].

[6] T. Yanagida, Horizontal symmetry and masses of neutrinos, in Proceedings of the workshop on unified theory and the baryon number of the universe, O. Sawada and A. Sugamoto eds., KEK, Tsukuba Japan, (1979), pg. 95 [INSPIRE].

[7] M. Gell-Mann, P. Ramond and R. Slansky, Complex spinors and unified theories, in Supergravity, P. van Nieuwenhuizen and D. Freedman eds., North Holland, Amsterdam The Netherlands, (1979), pg. 315 [Conf. Proc. C 790927 (1979) 315] [arXiv:1306.4669] [INSPIRE].

[8] S.L. Glashow, The challenge of underground physics, in Quarks and leptons, M. Lévy et al. eds., Plenum, New York U.S.A., (1980), pg. 707 [inSPIRE]. 
[9] R.N. Mohapatra and G. Senjanović, Neutrino mass and spontaneous parity violation, Phys. Rev. Lett. 44 (1980) 912 [INSPIRE].

[10] Z.-Z. Xing, Naturalness and testability of TeV seesaw mechanisms, Prog. Theor. Phys. Suppl. 180 (2009) 112 [arXiv:0905.3903] [INSPIRE].

[11] Z.-Z. Xing and S. Zhou, Multiple seesaw mechanisms of neutrino masses at the TeV scale, Phys. Lett. B 679 (2009) 249 [arXiv:0906.1757] [InSPIRE].

[12] R.N. Mohapatra and J.W.F. Valle, Neutrino mass and baryon number nonconservation in superstring models, Phys. Rev. D 34 (1986) 1642 [INSPIRE].

[13] N. Arkani-Hamed, L.J. Hall, H. Murayama, D. Tucker-Smith and N. Weiner, Small neutrino masses from supersymmetry breaking, Phys. Rev. D 64 (2001) 115011 [hep-ph/0006312] [INSPIRE].

[14] F. Borzumati and Y. Nomura, Low scale seesaw mechanisms for light neutrinos, Phys. Rev. D 64 (2001) 053005 [hep-ph/0007018] [inSPIRE].

[15] R. Kitano, Small Dirac neutrino masses in supersymmetric grand unified theories, Phys. Lett. B 539 (2002) 102 [hep-ph/0204164] [INSPIRE].

[16] R.L. Arnowitt, B. Dutta and B. Hu, Yukawa textures, neutrino masses and Hořava-Witten M-theory, Nucl. Phys. B 682 (2004) 347 [hep-th/0309033] [INSPIRE].

[17] S. Abel, A. Dedes and K. Tamvakis, Naturally small Dirac neutrino masses in supergravity, Phys. Rev. D 71 (2005) 033003 [hep-ph/0402287] [InSPIRE].

[18] P.Q. Hung, Brane world unification of quark and lepton masses and its implication for the masses of the neutrinos, Nucl. Phys. B 720 (2005) 89 [hep-ph/0412262] [INSPIRE].

[19] P. Ko, T. Kobayashi and J.-H. Park, Lepton masses and mixing angles from heterotic orbifold models, Phys. Rev. D 71 (2005) 095010 [hep-ph/0503029] [INSPIRE].

[20] C. Hagedorn and W. Rodejohann, Minimal mass matrices for Dirac neutrinos, JHEP 07 (2005) 034 [hep-ph/0503143] [INSPIRE].

[21] M. Lindner, M. Ratz and M.A. Schmidt, Renormalization group evolution of Dirac neutrino masses, JHEP 09 (2005) 081 [hep-ph/0506280] [INSPIRE].

[22] R. Friedberg and T.D. Lee, A possible relation between the neutrino mass matrix and the neutrino mapping matrix, HEPNP 30 (2006) 591 [hep-ph/0606071] [INSPIRE].

[23] Z.-Z. Xing and H. Zhang, Distinguishable RGE running effects between Dirac neutrinos and Majorana neutrinos with vanishing Majorana CP-violating phases, Commun. Theor. Phys. 48 (2007) 525 [hep-ph/0601106] [INSPIRE].

[24] S. Luo, Z.-Z. Xing and X. Li, Generic Friedberg-Lee symmetry of Dirac neutrinos, Phys. Rev. D 78 (2008) 117301 [arXiv:0809.0781] [INSPIRE].

[25] N. Memenga, W. Rodejohann and H. Zhang, $A_{4}$ flavor symmetry model for Dirac neutrinos and sizable $U_{e 3}$, Phys. Rev. D 87 (2013) 053021 [arXiv:1301.2963] [INSPIRE].

[26] X.-W. Liu and S. Zhou, Texture zeros for Dirac neutrinos and current experimental tests, Int. J. Mod. Phys. A 28 (2013) 1350040 [arXiv:1211.0472] [InSPIRE].

[27] C.-S. Chen and L.-H. Tsai, Peccei-Quinn symmetry as the origin of Dirac neutrino masses, Phys. Rev. D 88 (2013) 055015 [arXiv:1210.6264] [INSPIRE]. 
[28] G.-J. Ding and Y.-L. Zhou, Dirac neutrinos with $S_{4}$ flavor symmetry in warped extra dimensions, Nucl. Phys. B 876 (2013) 418 [arXiv: 1304.2645] [INSPIRE].

[29] A. Aranda, C. Bonilla, S. Morisi, E. Peinado and J.W.F. Valle, Dirac neutrinos from flavor symmetry, Phys. Rev. D 89 (2014) 033001 [arXiv:1307.3553] [INSPIRE].

[30] S. Chakdar, K. Ghosh and S. Nandi, A predictive model of Dirac neutrinos, Phys. Lett. B 734 (2014) 64 [arXiv:1403.1544] [INSPIRE].

[31] A. Esmaili and A. Yu. Smirnov, Discrete symmetries and mixing of Dirac neutrinos, Phys. Rev. D 92 (2015) 093012 [arXiv:1510.00344] [INSPIRE].

[32] J. Zhang and S. Zhou, Relic right-handed Dirac neutrinos and implications for detection of cosmic neutrino background, Nucl. Phys. B 903 (2016) 211 [arXiv:1509.02274] [inSPIRE].

[33] S. Kanemura, K. Sakurai and H. Sugiyama, Probing models of Dirac neutrino masses via the flavor structure of the mass matrix, Phys. Lett. B 758 (2016) 465 [arXiv:1603.08679] [INSPIRE].

[34] Y. Fujimoto, K. Hasegawa, T. Nagasawa, K. Nishiwaki, M. Sakamoto and K. Tatsumi, Active Dirac neutrinos via SU(2) $)_{L}$ doublets in 5d, JHEP 06 (2016) 178 [arXiv:1601.05265] [INSPIRE].

[35] J.W.F. Valle and C.A. Vaquera-Araujo, Dynamical seesaw mechanism for Dirac neutrinos, Phys. Lett. B 755 (2016) 363 [arXiv:1601.05237] [INSPIRE].

[36] E. Ma and O. Popov, Pathways to naturally small Dirac neutrino masses, Phys. Lett. B 764 (2017) 142 [arXiv: 1609. 02538] [INSPIRE].

[37] D. Borah and A. Dasgupta, Naturally light Dirac neutrino in left-right symmetric model, JCAP 06 (2017) 003 [arXiv: 1702.02877] [INSPIRE].

[38] W. Wang and Z.-L. Han, Naturally small Dirac neutrino mass with intermediate $\mathrm{SU}(2)_{L}$ multiplet fields, arXiv:1611.03240 [INSPIRE].

[39] W. Wang, R. Wang, Z.-L. Han and J.-Z. Han, The B-L scotogenic models for Dirac neutrino masses, arXiv:1705.00414 [INSPIRE].

[40] W.H. Furry, On transition probabilities in double beta-disintegration, Phys. Rev. 56 (1939) 1184 [InSPIRE].

[41] S.M. Bilenky and C. Giunti, Neutrinoless double-beta decay: a probe of physics beyond the Standard Model, Int. J. Mod. Phys. A 30 (2015) 1530001 [arXiv:1411.4791] [inSPIRE].

[42] Z. Maki, M. Nakagawa and S. Sakata, Remarks on the unified model of elementary particles, Prog. Theor. Phys. 28 (1962) 870 [InSPIRE].

[43] B. Pontecorvo, Neutrino experiments and the problem of conservation of leptonic charge, Sov. Phys. JETP 26 (1968) 984 [Zh. Eksp. Teor. Fiz. 53 (1967) 1717] [INSPIRE].

[44] W. Grimus and L. Lavoura, $\mu-\tau$ interchange symmetry and lepton mixing, Fortsch. Phys. 61 (2013) 535 [arXiv:1207.1678] [InSPIRE].

[45] R.N. Mohapatra and C.C. Nishi, Implications of $\mu-\tau$ flavored CP symmetry of leptons, JHEP 08 (2015) 092 [arXiv:1506.06788] [INSPIRE].

[46] Z.Z. Xing and S. Zhou, Neutrinos in particle physics, astronomy and cosmology, Zhejiang University Press and Springer-Verlag, Berlin Germany, (2011) [INSPIRE]. 
[47] P.F. Harrison and W.G. Scott, $\mu-\tau$ reflection symmetry in lepton mixing and neutrino oscillations, Phys. Lett. B 547 (2002) 219 [hep-ph/0210197] [INSPIRE].

[48] I. Aizawa and M. Yasue, General property of neutrino mass matrix and CP-violation, Phys. Lett. B 607 (2005) 267 [hep-ph/0409331] [INSPIRE].

[49] T. Baba and M. Yasue, Majorana CP-violation in approximately $\mu-\tau$ symmetric models with $\operatorname{det}\left(M_{\nu}\right)=0$, Prog. Theor. Phys. 123 (2010) 659 [arXiv:1003.1438] [INSPIRE].

[50] Z.-Z. Xing and Y.-L. Zhou, A generic diagonalization of the $3 \times 3$ neutrino mass matrix and its implications on the $\mu-\tau$ flavor symmetry and maximal CP-violation,

Phys. Lett. B 693 (2010) 584 [arXiv:1008.4906] [INSPIRE].

[51] Z.-Z. Xing and Z.-H. Zhao, A review of $\mu-\tau$ flavor symmetry in neutrino physics, Rept. Prog. Phys. 79 (2016) 076201 [arXiv:1512.04207] [InSPIRE].

[52] S. Luo and Z.-Z. Xing, Resolving the octant of $\theta_{23}$ via radiative $\mu-\tau$ symmetry breaking, Phys. Rev. D 90 (2014) 073005 [arXiv: 1408.5005] [InSPIRE].

[53] Z.-Z. Xing and J.-Y. Zhu, Neutrino mass ordering and $\mu-\tau$ reflection symmetry breaking, arXiv:1707.03676 [INSPIRE].

[54] B. Adhikary, A. Ghosal and P. Roy, $\mu-\tau$ symmetry, tribimaximal mixing and four zero neutrino Yukawa textures, JHEP 10 (2009) 040 [arXiv: 0908.2686] [INSPIRE].

[55] Z.-H. Zhao, On the breaking of $\mu-\tau$ flavor symmetry, arXiv: 1605.04498 [INSPIRE].

[56] Z.-H. Zhao, Breakings of the neutrino $\mu-\tau$ reflection symmetry, JHEP 09 (2017) 023 [arXiv: 1703.04984] [INSPIRE].

[57] Z.-C. Liu, C.-X. Yue and Z.-H. Zhao, Neutrino $\mu-\tau$ reflection symmetry and its breaking in the minimal seesaw, JHEP 10 (2017) 102 [arXiv:1707.05535] [INSPIRE].

[58] T.P. Cheng, E. Eichten and L.-F. Li, Higgs phenomena in asymptotically free gauge theories, Phys. Rev. D 9 (1974) 2259 [INSPIRE].

[59] M.E. Machacek and M.T. Vaughn, Two loop renormalization group equations in a general quantum field theory 2. Yukawa couplings, Nucl. Phys. B 236 (1984) 221 [INSPIRE].

[60] B. Grzadkowski and M. Lindner, Nonlinear evolution of Yukawa couplings, Phys. Lett. B 193 (1987) 71 [inSPIRE].

[61] V.D. Barger, M.S. Berger and P. Ohmann, Supersymmetric grand unified theories: two loop evolution of gauge and Yukawa couplings, Phys. Rev. D 47 (1993) 1093 [hep-ph/9209232] [INSPIRE].

[62] C.-W. Chiang, CP violation for leptons at higher energy scales, Phys. Rev. D 63 (2001) 076009 [hep-ph/0011195] [INSPIRE].

[63] M. Lindner, M. Ratz and M.A. Schmidt, Renormalization group evolution of Dirac neutrino masses, JHEP 09 (2005) 081 [hep-ph/0506280] [INSPIRE].

[64] Z.-Z. Xing, A novel parametrization of $\tau$-lepton dominance and simplified one-loop renormalization-group equations of neutrino mixing angles and CP-violating phases, Phys. Lett. B 633 (2006) 550 [hep-ph/0510312] [INSPIRE].

[65] T. Ohlsson and S. Zhou, Renormalization group running of neutrino parameters, Nature Commun. 5 (2014) 5153 [arXiv:1311.3846] [INSPIRE]. 
[66] Z.-Z. Xing, H. Zhang and S. Zhou, Updated values of running quark and lepton masses, Phys. Rev. D 77 (2008) 113016 [arXiv:0712.1419] [InSPIRE].

[67] Z.-Z. Xing, H. Zhang and S. Zhou, Impacts of the Higgs mass on vacuum stability, running fermion masses and two-body Higgs decays, Phys. Rev. D 86 (2012) 013013 [arXiv:1112.3112] [INSPIRE].

[68] C. Jarlskog, Commutator of the quark mass matrices in the standard electroweak model and a measure of maximal CP-violation, Phys. Rev. Lett. 55 (1985) 1039 [InSPIRE].

[69] Z.-Z. Xing, Flavor mixing and CP-violation of massive neutrinos, Int. J. Mod. Phys. A 19 (2004) 1 [hep-ph/0307359] [InSPIRE].

[70] F. Capozzi, E. Di Valentino, E. Lisi, A. Marrone, A. Melchiorri and A. Palazzo, Global constraints on absolute neutrino masses and their ordering, Phys. Rev. D 95 (2017) 096014 [arXiv: 1703.04471] [INSPIRE].

[71] P.F. de Salas, D.V. Forero, C.A. Ternes, M. Tortola and J.W.F. Valle, Status of neutrino oscillations 2017, arXiv:1708.01186 [INSPIRE].

[72] Z.-Z. Xing, Implications of the Daya Bay observation of $\theta_{13}$ on the leptonic flavor mixing structure and CP-violation, Chin. Phys. C 36 (2012) 281 [arXiv:1203.1672] [INSPIRE].

[73] S. Antusch, J. Kersten, M. Lindner, M. Ratz and M.A. Schmidt, Running neutrino mass parameters in see-saw scenarios, JHEP 03 (2005) 024 [hep-ph/0501272] [INSPIRE].

[74] Y.-L. Zhou, $\mu-\tau$ reflection symmetry and radiative corrections, arXiv:1409.8600 [INSPIRE].

[75] W. Rodejohann and X.-J. Xu, Trimaximal $\mu-\tau$ reflection symmetry, Phys. Rev. D 96 (2017) 055039 [arXiv: 1705.02027] [INSPIRE].

[76] T2K collaboration, K. Abe et al., Measurement of neutrino and antineutrino oscillations by the $T 2 K$ experiment including a new additional sample of $\nu_{e}$ interactions at the far detector, arXiv: 1707.01048 [INSPIRE].

[77] M. Hartz, T2K neutrino oscillation results with data up to 2017 summer, talk given at the KEK colloquium, 4 August 2017. 